\title{
Collateral, DeFAult PENALties AND INFINite HORIZON
}

\section{EQUILIBRIUM}

\section{Mário Rui Páscoa ${ }^{a, *}$ and Abdelkrim Seghir ${ }^{b}$.}

a. Nova School of Business and Economics, 1099-032 Lisboa, Portugal.

b. The American University in Cairo, School of Business, AUC Avenue, P.O. Box 74 New Cairo 11835, Egypt.

\begin{abstract}
Páscoa and Seghir (2009) noticed that when collateralized promises become subject to utility penalties on default, Ponzi schemes may occur. However, equilibrium exists in some interesting cases. Under low penalties, equilibrium exists if the collateral does not yield utility (for example, when it is a productive asset or a security). Equilibrium exists also under more severe penalties and collateral utility gains, when the promise or the collateral are nominal assets and the margin requirements are endogenous: relative inflation rates and margin coefficients can make the income effects dominate the penalty effects. An equilibrium refinement avoids no-trade equilibria with unduly repayment beliefs. Our refinement differs from the one used by Dubey, Geanakoplos and Shubik (2005) as it does not eliminate no trade equilibria whose low delivery rates are consistent with the propensity to default of agents that are on the verge of selling.
\end{abstract}

Keywords: Incomplete markets, Default, Collateral, Utility Penalties, Ponzi Schemes.

JEL CLASSIFICATION: D52, D91.

\section{INTRODUCTION}

Ponzi schemes and utility penalties

The modern general equilibrium literature on default evolved mainly from two seminal contributions, the Dubey, Geanakoplos and Shubik (2005) paper on utility penalties and the Geanakoplos and Zame (1995) work on collateral. In an infinite horizon set-up, collateral has the beauty of eliminating Ponzi schemes, as shown by Araujo, Páscoa and Torres-Martínez (2002). However, when combined with a utility penalty, collateral might not avoid a Ponzi game, as illustrated by the examples in Páscoa and Seghir (2009) for utility penalties prohibiting full default. This observation was clarified recently by Martins-da-Rocha and Vailakis (2010), who noticed that, in these complete markets examples, a trivial no-trade equilibrium could nevertheless be found by setting the delivery rate at the minimal level,

\footnotetext{
${ }^{*}$ Corresponding author. E-Mail address: pascoa@novasbe.pt. Phone: +351.213.80.16.00, Fax: +351.213 .88 .60 .73 . Support from a Nova Forum grant is gratefully acknowledged.
} 
even though such unduly expectation about the delivery rate is not consistent with the harsh penalty. Once the equilibrium is refined, along the lines of the refinement in Dubey, Geanakoplos and Shubik (2005), as proposed by Martins da Rocha and Vailakis (2010), non-existence of equilibrium prevails for the harsh penalties in these examples. Given this negative observation, can we assert anything about existence of infinite horizon equilibrium when promises are secured by collateral but subject to utility penalties on default? These penalties try to capture reputation consequences or the embarrassment when asking for new loans, which may be observed in some credit markets.

Páscoa and Seghir (2009) proposed a default punishment bounded by the utility from individual endowments, for borrowed amounts that could be collateralized by aggregate physical resources. However, as Martins-da-Rocha and Vailakis (2010) pointed out, for other borrowing plans, penalties may be big and consumers do not replace Ponzi schemes by default. Hence, if agents are just constrained by their budget restrictions, what can be said? Actually, this is an important issue since, in infinite horizon economies, it is not possible to show that an equilibrium for the economy with explicit aggregate resources bounds is also an equilibrium for the economy where agents are just budget-constrained.

\section{Existence results}

In the current paper we present some existence results. As in Dubey, Geanakoplos and Shubik (2005), penalties are proportional to the real default, using a reference bundle to compute the price index. We find an upper bound on penalty coefficients that make the collateral cost never fall below the promise price, which was the crucial condition for the absence of Ponzi schemes in Araujo, Páscoa and Torres-Martínez (2002). The upper bound on penalty coefficients turns out to be the minimum of the marginal utility along the direction of the reference bundle, over the set of feasible bundles. Under this upper bound, Ponzi schemes are ruled out, but, in the presence of penalties, this is only necessary, but not sufficient, for existence of equilibrium. Consumers may use utility gains from collateral consumption to do a generalized form of a Ponzi scheme with reallocations of expenditures at every node. Existence of equilibrium is, therefore, guaranteed, under these moderate penalties, provided that the collateral does not give any utility, as when it is a durable commodity with no utility yields (as in Fostel and Geanakoplos (2008)), a productive asset or a share in it (as in Kubler and Schmedders (2003)) or any real security in positive net supply that cannot be short-sold. Otherwise, additional constraints had to be added. Actually, the default punishments that utility penalties try to capture are more often observed in collateralized borrowing for the purchase of equipment or securities (for the former, default insurance is usually required and for the latter default triggers personal bankruptcy). In contrast, in mortgages, when foreclosure occurs, the debtor can have a fresh start.

Moderate penalties and absence of direct utility gains from collateral may be a sufficient condition for existence of equilibrium but it is not necessary. Moderate penalties is a strong condition as it makes agents give full default (as in the model where utility penalties were absent). However, equilibrium is compatible with partial default or no default. We give an example motivating our second type of results. In this example, the sum, across next nodes, of the marginal penalty effects is dominated by the sum of the marginal income effects, even when at some nodes the penalty allows for a partial default or prevents default. The problem is that this outcome depends on relative prices and there is, in general, no room to choose relative spot prices as these are already pinned down by market clearing in commodity markets. There is, nevertheless, an interesting case where there are degrees of freedom in market clearing prices. It is the case where contracts are nominal, more precisely, when the promise, the collateral or both are nominal assets (that is, have exogenous yields in units of account). 
For example, when the contract is a loan with exogenous obligations (hence, nominal) that has the purpose of financing the purchase of a bond or a stock (hence, a nominal or a real asset, respectively). In both cases, there is indeterminacy with respect to the inflation rates at the next nodes. In the latter, high inflation rates across all the next nodes, devaluate the promised payments but not the collateral (and, therefore, reduce the real value of default on which the penalty is applied). In the former, there are trade-offs among the inflation rates, but for, endogenous margin requirements, we can make the marginal penalty effects become dominated by the marginal income effects (and generalized Ponzi schemes can not occur).

\section{Refinement of equilibrium}

Do the equilibria that we found resist a refinement that eliminates no-trade outcomes with spurious delivery beliefs? Dubey, Geanakoplos and Shubik (2005) showed that for unsecured promises subject to utility penalties on default, a trivial equilibrium always exists by setting promises prices, delivery rates and financial trades equal to zero. But the expectations about delivery rates in this trivial equilibrium may be spurious, when penalty coefficients exceed marginal utilities of income. In fact, in this case, agents are strictly conscientious and we should have instead an expectation of full delivery. To rule out this trivial outcome, with unduly pessimistic beliefs, the authors proposed a refinement: checking whether the equilibrium is a limit of equilibrium of economies where the government buys and sells an arbitrarily small amount of the promise, with full delivery. In the case of secured promises, the natural candidate for a trivial equilibrium would be one where promises are not traded and deliveries rates are at the lowest possible level, given by the ratio of the minimal delivery (the minimum between the promise and the collateral) to the promised one. In complete markets, such equilibrium can be trivially found, but that is not the case under incomplete markets. Nevertheless, we may want to rule out unduly pessimistic expectations, but not by using this refinement, as it tends to eliminate too many no-trade finite horizon equilibria (possibly ones with duly low delivery rates and duly low promise prices, whose infinite horizon cluster points would be incompatible with generalized Ponzi schemes).

The refinement should be as effective in eliminating unduly optimistic expectations as it is in eliminating unduly pessimistic expectations. It should check, in the case promises are not traded, whether the expectations about delivery rates are consistent with the default attitude (the relation between penalty coefficients and marginal utilities of income) of agents on the verge of selling. These are the agents that have their Kuhn-Tucker conditions with respect to selling already holding as equalities and are already purchasing some amount of what can serve as collateral. If, at an equilibrium, there are such agents, we check if the equilibrium can be approximated by equilibria of economies where the government buys a small amount of the promise and uses lump-sum taxes today and lump-sum subsidies tomorrow, to induce small sales, so that the delivery rates in these auxiliary economies are consistent with the default attitude of true agents (those that were on the verge of selling). We show that our proposed refinement is still effective in eliminating no-trade equilibria with unduly pessimistic expectations when agents are strictly conscientious, but does not eliminate no-trade equilibria with duly low expectations. In particular, we show that the equilibria we found, under moderate penalties or when contracts are nominal, are refined equilibria.

The paper is organized as follows. The next section identifies the general condition under which a cluster point of finite horizon equilibria will be an infinite horizon equilibrium. Then, in Section 3, we see that, when this condition fails, infinite-lived agents can do a generalized form of a Ponzi scheme. In Section 4 we address moderate penalties and in Section 5 we look at nominal contracts. Finally, in 
Section 6 we propose a new refinement procedure.

\section{FROM FINITE HORIZON EQUILIBRIA TO INFINITE HORIZON EQUILIBRIA.}

Consumers trade collateralized promises over a countably infinite tree D with finitely many branches at each node. Let $\mathcal{T}=\{0,1, \ldots\}$ be the set of dates and $\xi_{0}$ be the root of the tree $D$. Given a node $\xi \in D$, let $t(\xi) \in \mathcal{T}$ be the date of node $\xi$. We write $\xi>\xi^{\prime}$ if $t(\xi)>t\left(\xi^{\prime}\right)$ and $\xi^{\prime}$ belongs to the event-tree that starts at $\xi$. Let $\xi^{+}$be the set of immediate successors of $\xi$.

At each node $\xi$ a finite number $G$ of durable or perishable goods is traded together with a finite set $J(\xi)$ of one-period promises. Short sales of promises are secured by collateral. We want to allow for the collateral to be not necessarily a durable good, but also a productive asset or a security in positive net supply that pays real returns and cannot be short sold (as in Kubler and Schmedders (2003)). This can be accommodated by treating securities formally as durable goods that do not yield utility. In this context, we may have a non-diagonal transformation matrix $Y(\xi)$, of type $G$. $G$, indicating how commodities of the previous node convert into commodities of the node $\xi$. If $g$ is a durable good, the column $(Y(\xi))^{g}$ is equal to $a(\xi, g) e_{g}$, where $a(\xi, g)$ is a depreciation factor. If $g$ is a security, $Y_{g g}(\xi)=1$ and $\left(Y_{g^{\prime} g}\right)_{g^{\prime} \neq g}$ is a non-null vector of non-negative dividends. We will allow also for productive assets (as in Kubler and Schmedders (2003)) which can be treated formally as durable goods whose non-null columns in $Y(\xi)$ matrices represent their productive returns on other commodities.

There are $I$ consumers whose endowments and preferences verify the following assumptions.

Assumption $[\mathbf{E}]$. Endowments of consumer $i$ of commodity $g$ at node $\xi$, denoted by $\omega^{i}(\xi, g)$, satisfy

(i) $\exists W \in \mathbb{R}_{++}: \forall i \in I, \forall \xi \in D, \sum_{g \in G} \omega^{i}(\xi, g) \leq W$.

(ii) $\omega\left(\xi_{0}\right) \gg 0$ and, for $\xi>\xi_{0}$ and any $g, \omega(\xi, g)>0$ whenever the $g$-th row of $Y(\xi)$ is null.

Let $Y_{\xi_{1}, \xi_{n}}=Y\left(\xi_{n}\right) Y\left(\xi_{n-1}\right) \ldots Y\left(\xi_{2}\right)$ for $\xi_{k+1} \in \xi_{k}{ }^{+}$(and equal to the identity matrix if $n=$ 1). The aggregate physical resources available at node $\xi$ are given by $\gamma_{\xi}=\sum_{i} W_{\xi}^{i}$, where $W_{\xi}^{i}=$ $\sum_{\eta \in\left\{\xi_{0}, \ldots, \xi^{-}, \xi\right\}} Y_{\eta, \xi} \omega^{i}(\eta)$.

Assumption [U]. $\forall i \in I$, preferences over consumption are described by a time and state separable utility $U^{i}$ with instantaneous utility $v_{\xi}^{i}: \mathbb{R}_{+}^{G} \longrightarrow \mathbb{R}$ which is continuous, monotone and concave with $v_{\xi}^{i}(0)=0$. Moreover, $\forall i \in I, \forall \alpha \in \mathbb{R}_{+}^{G}, \sum_{\xi \in D} v_{\xi}^{i}(\alpha)$ is finite. We assume also that $\sum_{\xi \in D} v_{\xi}^{i}\left(\gamma_{\xi}\right)<\infty .^{1}$

Consumers take as given prices $p$ for goods, prices $q$ for promises and delivery rates $K$ on the promises. A choice variable is a plan $(x, \theta, \varphi, \Delta)$ consisting of purchases of goods not for collateral purposes, promises purchases, promises short sales and repayments, respectively. As in Páscoa and Seghir (2009), budget constraints are given by:

$$
p\left(\xi_{0}\right) \cdot\left(x^{i}\left(\xi_{0}\right)-\omega^{i}\left(\xi_{0}\right)\right)+p\left(\xi_{0}\right) C\left(\xi_{0}\right) \varphi^{i}\left(\xi_{0}\right)+q\left(\xi_{0}\right) \cdot\left(\theta^{i}\left(\xi_{0}\right)-\varphi^{i}\left(\xi_{0}\right)\right) \leq 0,
$$

\footnotetext{
${ }^{1}$ When $Y$ was diagonal with elements uniformly bounded away from one, the assumptions made by Páscoa and Seghir (2009) in [E] (that endowments were uniformly bounded) and in [U] (that the utility of a bounded plan is finite) were sufficient to ensure $\sum_{\xi \in D} v_{\xi}^{i}\left(\gamma_{\xi}\right)<\infty$.
} 
and $\forall \xi \in D \backslash\left\{\xi_{0}\right\}$,

$$
\begin{aligned}
p(\xi) \cdot x^{i}(\xi) & +p(\xi) C(\xi) \varphi^{i}(\xi)+q(\xi) \cdot\left(\theta^{i}(\xi)-\varphi^{i}(\xi)\right) \leq p(\xi) \omega^{i}(\xi)+p(\xi) Y(\xi) x^{i}\left(\xi^{-}\right) \\
& +p(\xi) Y(\xi) C\left(\xi^{-}\right) \varphi^{i}\left(\xi^{-}\right)+\sum_{j \in J\left(\xi^{-}\right)} K^{j}(\xi) p(\xi) A^{j}(\xi) \theta_{j}^{i}\left(\xi^{-}\right)-\sum_{j \in J\left(\xi^{-}\right)} \Delta_{j}^{i}(\xi),
\end{aligned}
$$

To shorten the notations, we define $\operatorname{MIN}_{j}(\xi)=\min \left\{p(\xi) A^{j}(\xi), p(\xi) Y(\xi) C^{j}\left(\xi^{-}\right)\right\}$, for each node $\xi$ and for each asset $j \in J\left(\xi^{-}\right)$. The minimal repayment constraint requires

$$
\operatorname{MIN}_{j}(\xi) \varphi_{j}(\xi) \leq \Delta_{j}(\xi) .
$$

The coefficients of the utility penalty, linear on default, are given by $\tilde{\lambda}_{j}^{i}(\xi)=\frac{\lambda_{j}^{i}(\xi)}{p(\xi) b(\xi)}$, where $b(\xi)$ is a reference bundle. So, the entire payoff function of consumer $i$ is

$$
\Pi^{i}\left(x^{i}, \theta^{i}, \varphi^{i}, \Delta^{i}\right):=\sum_{\xi \in D} v_{\xi}^{i}\left(\tilde{x}^{i}(\xi)\right)-\sum_{\xi \in D \backslash\{0\}} \sum_{j \in J\left(\xi^{-}\right)} \lambda_{j}^{i}(\xi) \frac{\left[p(\xi) A^{j}(\xi) \varphi_{j}^{i}\left(\xi^{-}\right)-\Delta_{j}^{i}(\xi)\right]^{+}}{p(\xi) b(\xi)},
$$

where

$\tilde{x}^{i}(\xi)=x^{i}(\xi)+C(\xi) \varphi^{i}(\xi)$ and $\left[p(\xi) A^{j}(\xi) \varphi_{j}^{i}\left(\xi^{-}\right)-\Delta_{j}^{i}(\xi)\right]^{+}=\max \left\{p(\xi) A^{j}(\xi) \varphi_{j}^{i}\left(\xi^{-}\right)-\Delta_{j}^{i}(\xi), 0\right\}$.

Definition 2.1 An equilibrium of $\mathcal{E}$ is a vector $\left(\bar{p}, \bar{q}, \bar{K},\left(\bar{x}^{i}, \bar{\theta}^{i}, \bar{\varphi}^{i}, \bar{\Delta}^{i}\right)_{i \in I}\right)$ such that $\bar{p}(\xi)>0$ at any node $\xi \in D$ and verifying:

(i) For each agent $i \in I,\left(\bar{x}^{i}, \bar{\theta}^{i}, \bar{\varphi}^{i}, \bar{\Delta}^{i}\right) \in \operatorname{argmax} \Pi^{i}(x, \theta, \varphi, \Delta)$ subject to budget and minimal repayment constraints.

(ii) $\sum_{i \in I}\left[\bar{x}^{i}\left(\xi_{0}\right)+C\left(\xi_{0}\right) \bar{\varphi}^{i}\left(\xi_{0}\right)\right]=\sum_{i \in I} \omega^{i}\left(\xi_{0}\right)$,

(iii) $\sum_{i \in I}\left[\bar{x}^{i}(\xi)+C(\xi) \bar{\varphi}^{i}(\xi)\right]=\sum_{i \in I}\left[\omega^{i}(\xi)+Y(\xi) \bar{x}^{i}\left(\xi^{-}\right)+Y(\xi) C\left(\xi^{-}\right) \bar{\varphi}^{i}\left(\xi^{-}\right)\right], \quad \forall \xi \in D \backslash\{0\}$,

(iv) $\sum_{i \in I} \bar{\theta}^{i}=\sum_{i \in I} \bar{\varphi}^{i}$

(v) $\forall \xi \in D \backslash\left\{\xi_{0}\right\}, \forall j \in J\left(\xi^{-}\right), \bar{K}^{j}(\xi) \sum_{i \in I} \bar{p}(\xi) A^{j}(\xi) \bar{\theta}_{j}^{i}\left(\xi^{-}\right)=\sum_{i \in I} \bar{\Delta}_{j}^{i}(\xi)$.

Recall that in an economy with a truncated, finite, horizon $T$, the Kuhn-Tucker conditions on $\Delta_{j}^{i}(\xi), \varphi_{j}^{i}(\xi), \theta_{j}^{i}(\xi), x(\xi, g)$ require the existence of non-negative multipliers $\mu^{i}(\xi)$ and $\rho_{j}^{i}(\eta)$ for $\eta \in \xi^{+}$, together with $v_{\xi}^{i^{\prime}}(\tilde{x}(\xi)) \in \partial v_{\xi}^{i}\left(x^{i}(\xi)+C(\xi) \varphi(\xi)\right)$ and $d_{j}^{i}(\eta) \in[0,1]$ supergradient of the function $\max \{0, \cdot\}$ evaluated at $p(\eta) A^{j}(\eta) \varphi_{j}^{i}(\xi)-\Delta_{j}^{i}(\eta)$ such that:

$$
\begin{aligned}
& \tilde{\lambda}_{j}^{i}(\xi) d_{j}^{i}(\xi)+\rho_{j}^{i}(\xi)=\mu^{i}(\xi) \\
& \mu^{i}(\xi)\left(p(\xi) C^{j}(\xi)-q^{j}(\xi)\right)-v_{\xi}^{i^{\prime}}(\tilde{x}(\xi)) C^{j}(\xi) \geq \sum_{\eta \in \xi^{+}}\left[\mu^{i}(\eta)\left(p(\eta) Y(\eta) C^{j}(\eta)-\operatorname{MIN}_{j}(\eta)\right)\right. \\
& -\tilde{\lambda}_{j}^{i}(\eta) d_{j}^{i}(\eta)\left(p\left(\eta A^{j}(\eta)-\operatorname{MIN}_{j}(\eta)\right)\right] \\
& \mu^{i}(\xi) q^{j}(\xi) \geq \sum_{\eta \in \xi^{+}} \mu^{i}(\eta) K^{j}(\eta) p(\eta) A^{j}(\eta) \\
& \forall g \in G, \quad \mu^{i}(\xi) p(\xi, g) \geq v_{\xi}^{\prime}(\tilde{x}(\xi), g)+\sum_{\eta \in \xi^{+}} \mu^{i}(\eta) p(\eta)(Y(\xi))^{g},
\end{aligned}
$$


with equalities in (5), (6) or (7) holding when $\varphi_{j}(\xi)>0, \theta_{j}(\xi)>0$ or $x(\xi, g)>0$, respectively.

Equilibrium allocations $\bar{Z}^{i T} \equiv\left(x^{i T}, \theta^{i T}, \varphi^{i T}, \Delta^{i T}\right)$ of finite horizon economies have upper bounds, uniformly on the horizon $T$. Let us see that equilibrium prices and the associated equilibrium multiplies also have uniform upper bounds. We normalize prices by placing $(p(\xi), q(\xi))$ in the $G+J(\xi)-1$ dimensional simplex. The multipliers $\mu^{i}(\xi)$ and $\rho_{j}(\xi)$ have upper bounds that are independent of prices and of the terminal horizon $T$ of the economy, as established in Remark 1 in the Appendix. So, node by node, equilibrium variables (prices, delivery rates, allocations, multipliers and the above supergradients) of all finite horizon economies have common upper bounds.

Consider the sequence $\left(\bar{p}^{T}, \bar{q}^{T}, \bar{K}^{T},\left(\bar{Z}^{i T}, \mu^{i T}, \rho^{i T}, v^{i T^{\prime}}, d^{i T}\right)_{i}\right)$ of equilibrium prices, allocations, multipliers and supergradients (of the functions $v_{\xi}^{i}$ and $\max \{0, \cdot\}$ ) verifying the Kuhn-Tucker conditions, for the truncated economies. This sequence has, node by node, a cluster point $\left(p, q, K,\left(\bar{Z}^{i}, \mu^{i}, \rho^{i}, v^{i^{\prime}}, d^{i}\right)_{i}\right)$. Observe that at the price cluster point $p$ the payoff functions are well defined, as $p(\xi)>0$ at any node $\xi$, by Lemma 3 in the Appendix.

Proposition. An equilibrium exists for the infinite horizon economy if

$$
\limsup _{T} \sum_{\xi: t(\xi)=T}\left(v_{\xi}^{i^{\prime}}\left(\bar{Z}^{i}\right) C(\xi)-\mu^{i}(\xi)(p(\xi) C(\xi)-q(\xi))\right) \varphi(\xi) \leq 0,
$$

for any short sales trajectory $\varphi$ which is part of a plan $Z=(x, \theta, \varphi, \Delta)$ satisfying budget and minimal repayment constraints at prices and delivery rates $(p, q, K)$.

Proof. See Appendix.

In the next section we interpret the failure of condition (8) as an opportunity for doing a generalized version of a Ponzi squeme. Then, in Sections 4 and 5 we present existence results in contexts where condition (8) holds.

Remark: Páscoa and Seghir (2009) assumed that, for each node $\xi$ and each agent $i$,

$$
\tilde{\lambda}_{j}^{i}(\eta)\left[p(\eta) A^{j}(\eta)-\operatorname{MIN}_{j}(\eta)\right] \varphi_{j}^{i}(\xi)<v^{i}\left(\omega^{i}(\eta)\right), \forall \eta \in \xi^{+},
$$

whenever

$$
C^{j}(\xi) \varphi_{j}^{i}(\xi) \leq \sum_{i} W^{i}(\xi)
$$

This assumption does not suffice to get existence of equilibrium in infinite horizon economies, as for short sales plans that do not verify (10) nothing is being said about the penalty. ${ }^{2}$ However, if (10) is being added as a short sales constraint or if (9) alone had been imposed, then the condition in the Proposition would hold. In fact, by (5) $\lim \sup _{T} \sum_{\xi: t(\xi)=T}\left(v_{\xi}^{i^{\prime}}\left(\bar{Z}^{i}\right) C(\xi)-\mu^{i}(\xi)(p(\xi) C(\xi)-q(\xi))\right) \varphi(\xi)<$ $\lim \sup _{T} \sum_{\xi: t(\xi)=T} v^{i}\left(\omega^{i}\right)$. The desired condition holds as $U^{i}\left(\omega^{i}\right)<\infty$.

\section{Generalized Ponzi schemes.}

Note that in the absence of penalties, condition (8) in the Proposition is clearly satisfied due to (5). However, when there are penalties, this condition may fail even when $p(\xi) C^{j}(\xi) \geq q^{j}(\xi)$. That is,

\footnotetext{
${ }^{2}$ In the existence proof provided by Páscoa and Seghir (2009), it was overlooked (in the last sentence of the proof) that the short sales $\hat{\varphi}$ of the alternative plan might not fulfil $C^{j}(\xi) \hat{\varphi}^{j}(\xi) \leq \sum_{i} W^{i}(\xi)$.
} 
absence of Ponzi schemes (ensured by $p(\xi) C^{j}(\xi) \geq q^{j}(\xi)$ ) is not enough to guarantee existence of equilibrium. Agents could be doing what we will call a generalized Ponzi scheme, reallocating expenditures at each node $\xi$ towards some promise $j(\xi)$ with $v_{\xi}^{i^{\prime}} C^{j(\xi)}(\xi)-\mu^{i}(\xi)\left(p(\xi) C^{j(\xi)}(\xi)-q^{j(\xi)}(\xi)\right)>0$, so that the penalty and the minimal repayment associated with the increment in the short position in $j\left(\xi^{-}\right)$ are outweighed by the utility gain $v_{\xi}^{i \prime} C^{j(\xi)}(\xi)$ net of the utility $\operatorname{loss} \mu^{i}(\xi)\left(p(\xi) C^{j(\xi)}(\xi)-q^{j(\xi)}(\xi)\right)$ from reallocating expenditures (cutting in direct purchases of goods, for instance).

More precisely, a Ponzi scheme stricto sensu, consists in increasing the short position in promise $j(\sigma)$ at node $\sigma$ and then accommodate this by increasing the short position in promise $j(\xi)$ at the following nodes $\xi>\sigma$. According to Páscoa and Seghir (2009), Section 4.1, a Ponzi scheme exists if there is a direction $y=\left(y_{1}, y_{2}\right)$ consisting of a direction $y_{1}$ of changes in the short sales plan and a direction $y_{2}$ of changes in deliveries, given by:

$$
\left(y_{1}(\xi), y_{2}(\xi)\right)=\left(\alpha_{j(\xi)} e_{j(\xi)}, \alpha_{j\left(\xi^{-}\right)} p(\xi) A_{\xi}^{j\left(\xi^{-}\right)} e_{j\left(\xi^{-}\right)}\right)
$$

satisfying, for some $\sigma \in D, \alpha_{j(\xi)}=0$ for $\xi<\sigma$ and $\alpha_{j(\xi)}>0$ otherwise, verifying:

(a) $\alpha_{j(\sigma)}\left(p(\sigma) C^{j(\sigma)}(\sigma)-q^{j(\sigma)}(\sigma)\right)<0$, and

(b) $\alpha_{j(\xi)}\left(p(\xi) C^{j(\xi)}(\xi)-q^{j(\xi)}(\xi)\right)<\alpha_{j\left(\xi^{-}\right)} p(\xi)\left(Y(\xi) C^{j\left(\xi^{-}\right)}\left(\xi^{-}\right)-A^{j\left(\xi^{-}\right)}(\xi)\right)$, for $\xi>\sigma$.

This direction $y$ is right-admissible, with respect to constraints (1), (2) and (3). Moreover, the direction is payoff-improving for the right-hand side changes: $\Pi(x, \theta, \varphi, \Delta)<\Pi\left(x, \theta, \varphi+h y_{1}, \Delta+h y_{2}\right)$ for $h>0$. The existence of such a direction follows when $p(\xi) C^{j(\xi)}(\xi)-q^{j(\xi)}(\xi)<0$ not just at node $\sigma$ but also at every $\xi>\sigma$ (by choosing $\alpha_{j(\xi)} / \alpha_{j\left(\xi^{-}\right)}$high enough, when the right-hand side in (b) is negative).

There is nevertheless an extended form of Ponzi schemes, compatible with $p(\xi) C^{j(\xi)}(\xi)-q^{j(\xi)}(\xi) \geq 0$ for any asset $j$ and at any node $\xi$. The cost of the joint operation of constituting the collateral and short selling $\left(p(\xi) C^{j}(\xi)-q^{j}(\xi)\right)$ may be positive but can be accommodated (together with the penalties and repayments) by reallocating expenditures, in a way that increases utility.

We say that a generalized Ponzi scheme exists if there is a direction $\tilde{y}=\left(\tilde{y}_{1}, \tilde{y}_{2}\right)$ consisting in a direction $\tilde{y}_{1}$ of changes in the short sales plan and a direction $\tilde{y}_{2}$ of changes in deliveries given by:

$$
\left(\tilde{y}_{1}(\xi), \tilde{y}_{2}(\xi)\right)=\left(\alpha_{j(\xi)} e_{j(\xi)}, \alpha_{j\left(\xi^{-}\right)} \operatorname{MIN}_{j}(\xi) e_{j\left(\xi^{-}\right)}\right)
$$

satisfying $\alpha_{j(\xi)}=0$ for $\xi \leq \sigma$ and $\alpha_{j(\xi)}>0$ otherwise, verifying:

$\left(\mathrm{a}^{\prime}\right) \alpha_{j(\sigma)}\left[\mu^{i}(\sigma)\left(p(\sigma) C^{j(\sigma)}(\sigma)-q^{j(\sigma)}(\sigma)\right)-v_{\sigma}^{i \prime} C^{j(\sigma)}(\sigma)\right]<0$ and

$$
\begin{gathered}
\alpha_{j(\xi)}\left[\mu^{i}(\xi)\left(p(\xi) C^{j(\xi)}(\xi)-q^{j(\xi)}(\xi)\right)-v_{\xi}^{i^{\prime}} C^{j(\xi)}(\xi)\right] \\
<\quad \alpha_{j\left(\xi^{-}\right)}\left[\mu^{i}(\xi)\left(p(\xi) Y(\xi) C^{j\left(\xi^{-}\right)}-\operatorname{MIN}_{j}(\xi)\right)\right. \\
\left.-\quad \tilde{\lambda}^{j(\xi)}(\xi) d^{j\left(\xi^{-}\right)}(\xi)\left(p(\xi) A^{j\left(\xi^{-}\right)}(\xi)-\operatorname{MIN}_{j}(\xi)\right)\right], \text { for } \xi>\sigma .
\end{gathered}
$$

Now, $\tilde{y}$ itself might not be right-admissible with respect to $(1)$ and $(2)$. However, as $v_{\xi}^{i \prime} C^{j(\xi)}(\xi)>0$, it is right-payoff-improving even when we discount the utility loss $\mu^{i}(\xi)\left(p(\xi) C^{j(\xi)}(\xi)-q^{j(\xi)}(\xi)\right) \alpha_{j}(\xi)$ involved in reallocating expenditures so that budget constraints are satisfied ${ }^{3}$.

Now, the existence of such direction $\tilde{y}$ follows when $v_{\xi}^{i^{\prime}} C^{j(\xi)}(\xi)-\mu^{i}(\xi)\left(p(\xi) C^{j(\xi)}(\xi)-q^{j(\xi)}(\xi)\right)>0$ for some asset at each node $\xi \geq \sigma$ (this is immediate if the right-hand side in (b') is nonnegative, otherwise

\footnotetext{
${ }^{3}$ Say by cutting in a perishable commodity $g$ satisfying Inada's condition in the amount $\beta_{\xi} \equiv$ $\alpha_{j(\xi)}\left(p(\xi) C^{j(\xi)}(\xi)-q^{j(\xi)}(\xi)\right) / p(\xi, g)$ with marginal loss $\beta_{\xi} v_{g}^{i^{\prime}} \leq \mu^{i}(\xi)\left(p(\xi) C^{j(\xi)}(\xi)-q^{j(\xi)}(\xi)\right) \alpha_{j}(\xi)$.
} 
$\alpha_{j}(\xi)$ can be found high enough, relative to $\alpha_{j}\left(\xi^{-}\right)$so that (b') holds). Generalized Ponzi schemes are not scalable up by an arbitrarily large amount (unless the marginal utility were constant), contrary to what happened with Ponzi schemes, but if there is an opportunity for agent $i$ to do a generalized Ponzi scheme at $(\bar{Z}, p, q, K)$, cluster point of finite horizon equilibria, then $\bar{Z}^{i}$ is not optimal at $(p, q, K)$. Notice that the dominating short sales plan obtained by adding $h \tilde{y}_{1}$, with $h>0$, might not be bounded, even for $h$ arbitrarily small, as, node by node, $\alpha_{j}(\xi)$ may have to be sufficiently high relative to $\alpha_{j}\left(\xi^{-}\right)$.

Clearly, generalized Ponzi schemes are avoided when for any node $\xi$ utility penalties are moderate enough to guarantee $p(\xi) C(\xi) \geq q(\xi)$, and the collateral does not yield utility $\left(v_{\xi}^{i^{\prime}} C(\xi)=0\right)$, as in the cases of a collateral which is a security in positive net supply that cannot be sold short or a durable good that does not yield utility (as in Kubler and Schmedders (2003) and Fostel and Geanakoplos (2008)). Actually, utility penalties seem to be more relevant for promises backed by financial collateral than for mortgages, which tend to be non-recourse loans where just the durable goods-collateral is garnishable without further reputation or credit-access consequences when default occurs.

\section{Existence RESUlts.}

\subsection{Moderate penalties.}

We present a result that ensures that condition (8) in the Proposition holds. Let $r_{\xi}^{i}(b(\xi))$ be the minimum of the right derivative, $\delta^{+} v_{\xi}^{i}(z, b(\xi))$, of $v_{\xi}^{i}$ along the direction of the reference bundle $b(\xi)$, taken over all feasible bundles $z$ (see Lemma 2 in the Appendix).

\section{Theorem 1 (Moderate penalties)}

If $\lambda_{j}^{i}(\xi)<r_{\xi}^{i}(b(\xi))$, then $p(\xi) C(\xi) \geq q(\xi)$ in equilibrium of finite-horizon economies and Ponzi schemes, in stricto sensu, are avoided.

Equilibrium for the infinite-horizon economy exists if, in addition,

(a) the collateral does not yield utility (it is a durable good that does not give any utility or a productive asset or a security in positive net supply that cannot be short sold),

(b) or there is $\kappa \in(0,1)$ such that, for each agent $i$ and at each node $\xi, \omega^{i}(\xi) \geq \kappa W^{i}(\xi)$ and short sales plans $\varphi$ are required to be such that $\frac{p(\xi) C^{j}(\xi) \varphi_{j}(\xi)}{p(\xi) W^{i}(\xi)}$ is uniformly bounded.

(c) or new endowments $\omega^{i}(\xi)$ are uniformly bounded away from zero and short sales plans $\varphi$ are required to be collateralized by uniformly bounded bundles $C(\xi) \varphi(\xi)$ (in particular, when required to be collateralizable by bounded aggregate physical resources).

In (b) collateral is required not to explode faster (or tend to zero slower) than the consumer's cumulated resources. The value of the assets whose purchase is being financed by the secured loan cannot grow unboundedly, relatively to what the consumer estate is. In (c) the bound on collateral is exogenous or given by the aggregate resources in the economy. The assumption on endowments in (b) was previously used by Araujo, Páscoa and Torres-Martínez (2010), whereas the assumption on endowments made in (c) was considered by Magill and Quinzii (1996) and also by Araujo, Páscoa and Torres-Martínez (2010).

Under the hypothesis bounding penalty coefficients (but in the absence of (a), (b) or (c)), we can say that, at a cluster point of finite horizon equilibria, the opportunity of doing a generalized Ponzi scheme may occur only for agents who have (6) with strict inequality. In particular, those buying asset 
$j$ at node $\xi$ (at the cluster point or except in finitely many finite horizon economies) do not have that opportunity. This observation follows from the proof of Theorem 1 (see the Appendix).

\subsection{Equilibrium without full default.}

However, the above low penalties, implying full default when the promises are traded, are not necessary for equilibrium existence. Partial default or even full repayment are compatible with equilibrium and may occur under higher penalty coefficients. In fact, generalized Ponzi schemes are avoided when $\mu^{i}(\xi)\left(p(\xi) C^{j}(\xi)-q^{j}(\xi)\right)-v_{\xi}^{i^{\prime}} \cdot C^{j}(\xi) \geq 0, \forall i, \forall \xi$. By (5), it suffices to have

$$
\sum_{\eta \in \xi^{+}} \mu^{i}(\eta)\left(p(\eta) Y(\eta) C^{j}(\xi)-\operatorname{MIN}_{j}(\eta)\right) \geq \sum_{\eta \in \xi^{+}} \tilde{\lambda}_{j}^{i}(\eta) d_{j}^{i}(\eta)\left(p(\eta) A^{j}(\eta) \operatorname{MIN}_{j}(\eta)\right), \forall i, \forall \xi,
$$

where $d_{j}^{i}(\eta)$ satisfies (4). Actually, by (4), (11) holds if

$$
\sum_{\mu \in \xi^{+}} \mu^{i}(\eta)\left(p(\eta) Y(\eta) C^{j}(\eta)-p(\eta) A^{j}(\eta)\right) \geq 0
$$

is satisfied for all $i$ and for all $\xi$. Moreover, when the collateral does not yield utility gains, it is enough to have (11) (or (12)) satisfied, at each node $\xi$, for some agent $i(\xi)$, as this implies $p(\xi) C^{j}(\xi) \geq q^{j}(\xi)$.

The difficulty is that condition (12) depends on relative spot prices $p(\eta)$ and on the marginal utilities of income $\mu^{i}(\eta)$ and, in general, it is not possible to guarantee that the market clearing spot prices (and the induced multiplier $\mu^{i}$ ) are such that (12) is satisfied, for an arbitrary combination of returns $\left(A^{j}\right)$ and collateral yields $Y C^{j}$. Let us give, nevertheless, an example where (12) holds for arbitrary penalty coefficients. This example will motivate our next result.

\section{Example 1}

There are two infinite-lived agents, the event-tree has two branches at each node $\xi$ (up $\left(u_{\xi}\right)$ and down $\left.\left(d_{\xi}\right)\right)$. There is one consumption good and preferences are given by $U^{i}(Z)=\sum_{\xi \in D} \beta^{t(\xi)} \psi_{\xi}^{i} v_{\xi}^{i}\left(Z_{\xi}\right)$, where $v_{\xi}^{i}\left(Z_{\xi}\right)=Z_{\xi}$ and $\psi_{\xi}$ is the belief that agent $i$ attaches to moving to $\xi$ once $\xi^{-}$was attained $\left(\psi_{u_{\xi}}^{i}+\psi_{d_{\xi}}^{i}=\psi^{i}(\xi), \sum_{\xi: t(\xi)=t} \psi_{\xi}^{i}=1, \forall t\right)$. There is one promise paying in the above consumption good and using as collateral a real security (on a productive asset) that is short-lived but is issued (or endowed) at each node. Formally, this collateral instrument can be treated as a second commodity that transforms into the consumption good at the next date and then disappears. Denote by $a_{\xi}$ the promised returns and by $y_{\xi}$ the collateral yields. The collateral coefficient is $C_{\xi}=1$ and we normalize prices by taking the perishable consumption good $(g=1)$ as the numeraire. The reference bundle in the penalty function is $b(\xi)=(1,0)$ and the penalty is given by $\sum_{\xi \in D} \beta^{t(\xi)} \psi_{\xi}^{i} \delta_{\xi}\left[a_{\xi} \varphi\left(\xi^{-}\right)-\Delta(\xi)\right]^{+}$.

Given endowments $\omega^{i}(\xi)=\left(\omega_{1}^{i}(\xi), \omega_{1}^{i}(\xi)\right)$ of the consumption good and the collateral instrument, we write consumers' constraints as usual, denoting by $p(\xi)$ the collateral price and by $q(\xi)$ the promise price. Suppose $\psi_{u_{\xi}}^{(1)}=\psi_{d_{\xi}}^{(1)}=\frac{1}{2}^{t(\xi)+1}$, whereas $\psi_{u_{\xi}}^{(2)}=\frac{2}{3} \psi_{\xi}^{(2)}$ and $\psi_{d_{\xi}}^{(2)}=\frac{1}{3} \psi_{\xi}^{(2)}$. If $a_{u_{\xi}}=2, a_{d_{\xi}}=$ $1, y_{u_{\xi}}=1$ and $y_{u_{\xi}}=2, \forall \xi$, then (12) holds with equality for agent 1 (and therefore (11) holds for this agent, for any penalty coefficients $\delta_{\xi}^{(1)}$ ). For agent 2 , we assume $\delta_{\xi}^{(2)} \geq 1$ (i.e.: $\lambda_{\xi}^{(2)} \geq \mu_{\xi}^{(2)}, \forall \xi$ ) and we take $\rho^{(2)}(\xi)=0$ so that $d^{(2)}(\xi)=\frac{1}{\delta_{\xi}^{(2)}}$.

For $\delta_{\xi}^{(1)}=1$ (i.e.: $\left.\lambda_{\xi}^{(1)}=\mu_{\xi}^{(1)}\right) \forall \xi$, we see that $K_{u_{\xi}}=0.9, p(\xi)=q(\xi)=\frac{4.6}{3} \beta$ and $K_{d_{\xi}}=1$ satisfy (4) through (7), with agent 1 on-the-verge of selling and agent 2 on-the-verge of buying.

For $\delta_{\xi}^{(1)}>1$ (i.e.: $\left.\lambda_{\xi}^{(1)}>\mu_{\xi}^{(1)}\right) \forall \xi$, we see that $K_{u_{\xi}}=K_{d_{\xi}}=1$ and $p(\xi)=q(\xi)=\frac{5}{3} \beta$ satisfy $(4)$ through (7), with agent 1 on-the-verge of selling and agent 2 on-the-verge of buying. 
Taking $\omega_{\xi}^{(1)}=(1,0)$ and $\omega_{\xi}^{(2)}=(1, \Omega), \forall \xi$, let $\theta_{\xi}^{(2)}=\Omega, \varphi_{\xi}^{(1)}=\Omega, \theta_{\xi}^{(i)} \varphi_{\xi}^{(i)}=0$ and $x_{2 \xi}^{(i)}=0$ (no purchase of commodity 2 beyond what might be used as collateral). Then, for $\delta_{\xi}^{(1)}=1$, we obtain $\Delta_{u_{\xi}}^{(1)}=$ $0.9 a_{u_{\xi}} \Omega=1.8 \Omega, \Delta_{d_{\xi}}^{(1)}=a_{d_{\xi}} \Omega=\Omega$. Take $x_{1}^{(i)}(\xi)=\omega^{(i)}(\xi)+Y_{\xi} C\left(\xi^{-}\right) \varphi^{i}\left(\xi^{-}\right)-\Delta^{(i)}(\xi)+K(\xi) a_{\xi} \theta^{i}\left(\xi^{-}\right)$. Then, $x_{1 u_{\xi}}^{(1)}=1-0.8 \Omega, x_{1 d_{\xi}}^{(1)}=1+\Omega, x_{1 u_{\xi}}^{(2)}=1+1.8 \Omega, x_{1 d_{\xi}}^{(2)}=1+\Omega$. Market clearing follows $\left(\sum_{i} x^{i}(\xi)=\sum_{i} \omega^{i}(\xi)+y_{\xi} \Omega\right)$ and we assume $\Omega<1.25$ to obtain an equilibrium.

For $\delta_{\xi}^{(1)}>1, \forall \xi$ (that is $\left.\lambda_{\xi}^{(1)}=\mu_{\xi}^{(1)}\right) \forall \xi$ ), the equilibrium allocation is given by the same promise allocation, $\Delta_{u_{\xi}}^{(1)}=2 \Omega, \Delta_{d_{\xi}}^{(1)}=\Omega, x_{1 u_{\xi}}^{(1)}=1-\Omega, x_{1 d_{\xi}}^{(1)}=1+\Omega, x_{1 u_{\xi}}^{(2)}=1+2 \Omega, x_{1 d_{\xi}}^{(2)}=1+\Omega$. We assume in this case $\Omega<1$. We can accommodate $\delta_{\xi}^{(1)}=1$ or $\delta_{\xi}^{(1)}>1$ in equilibrium.

\subsection{Nominal contracts.}

The above example where both the promise and the collateral are numeraire assets, could be redone with both being nominal assets (say, the promise is a loan, with exogenous yields, whose purpose is the purchase of a bond). This leads us to study what happens when the promise or the collateral are nominal assets. In both cases, collateralized borrowing is not inflation proof. Depending on what the inflation rates are (across the set of nodes $\xi^{+}$), the negative marginal penalty effects $\left(\tilde{\lambda}_{j}^{i}(\eta) d_{j}^{i}(\eta)\left(p(\eta) A^{j}(\eta)-\operatorname{MIN}_{j}(\eta)\right)\right)$ may become dominated by the positive marginal income effects $\left(\mu^{i}(\eta)\left(p(\eta) Y(\eta) C^{j}(\xi)-\operatorname{MIN}_{j}(\eta)\right)\right)$. When that happens, there is no room for generalized Ponzi schemes (as (11) holds) and equilibrium exists.

The indeterminacy with respect to inflation rates, at finite horizon equilibria of economies with nominal promises or nominal collateral, may allow us to pick an equilibrium where (11) holds (and, therefore, condition (8) of the Proposition holds at the cluster point). ${ }^{4}$

As usual, given a promise with nominal returns $b_{\xi}^{j}$, we let $A^{j}(\xi)=\frac{b_{\xi}^{j}}{\sigma_{\xi}} \mathbb{I}$ where $\sigma_{\xi}$ stands for $\|p(\xi)\|_{1}$. Recall that for unsecured nominal assets, we had a homogeneity of commodity demanded with respect to $\left(\sigma_{\eta}\right)_{\eta \in \xi^{+}}$: if we multiply $\sigma_{\eta}$ by $\alpha>0, \forall \eta \in \xi^{+}$, and adjust the portfolio (multiplying by $\alpha$ ) and asset prices (dividing by $\alpha$ ), we can maintain the original bundle at the same relative spot prices. For that homogeneity to hold also for secured promises, the collateral coefficients had to be adjusted also (divided by $\alpha$ ). Actually, in the case of contracts where the promises or the collateral are nominal, it is harder to accept exogeneity of these coefficients, which are now margin requirements. We will allow next for margin requirements to be determined in equilibrium: for a promise $j$, backed by a nominal or real security $g(j)$ (or a productive asset), $C_{g(j)}^{j}(\xi)$ becomes an equilibrium variable.

When promise $j$ is nominal and its collateral $g(j)$ is real, the quotient set (for the equivalence relation induced by the above homogeneity) can be taken as the set $\left\{\left(\left(\sigma_{\eta}\right)_{\eta \in \xi^{+}}, C_{g(j)}^{j}(\xi)\right):\left(\sigma_{\eta}^{-1}\right)_{\eta \in \xi^{+}} \in\right.$ $\left.\Delta^{\# \xi^{+}-1}, C_{g(j)}^{j} \in \mathbb{R}_{++}\right\} .^{5}$

Similarly, for a nominal promise $j$ secured under margin requirements $C_{g(j)}^{j}$ by a nominal collateral $g(j)$, with exogenous yields, in units of account, $\tilde{y}_{\eta}^{g(j)}$, we let $Y_{\eta}^{g(j)}=\tilde{y}_{\eta}^{g(j)} \frac{1}{\sigma_{\eta}} \mathbb{I}$. The real allocation is preserved under the transformation:

$$
\left(\left(\sigma_{\eta}\right)_{\eta \in \xi^{+}}, q^{j}(\xi), C_{g(j)}^{j}(\xi), \theta^{j}(\xi), \varphi^{j}(\xi)\right) \longrightarrow\left(\alpha\left(\sigma_{\eta}\right)_{\eta \in \xi^{+}}, \frac{1}{\alpha} q^{j}(\xi), \frac{1}{\alpha} C_{g(j)}^{j}(\xi), \alpha \theta^{j}(\xi), \alpha \varphi^{j}(\xi)\right), \alpha>0,
$$
(as $Y_{\eta}^{g(j)}\left(\alpha \sigma_{\eta}\right)=\frac{1}{\alpha} Y_{\eta}^{g(j)}\left(\sigma_{\eta}\right)$ and $\left.A^{j}(\eta)\left(\alpha \sigma_{\eta}\right)=\frac{1}{\alpha} A^{j}(\eta)\left(\sigma_{\eta}\right)\right)$. The quotient set can be taken as before. ${ }^{6}$

\footnotetext{
${ }^{4}$ We are not interested in checking whether the degree of freedom in the choice of inflation rates implies real indeterminacy of equilibria (and of what degree). This would be another theme, facing the difficulties associated with the non-differentiability of the functions involved in the default decisions.

${ }^{5}$ Alternatively, we could had normalized $\left(\left(\sigma_{\eta}\right)_{\eta \in \xi^{+}}, \frac{1}{C_{g(j)}^{j}(\xi)}\right) \in \Delta^{\# \xi^{+}}$.

${ }^{6}$ For the less interesting case of a real promise backed by nominal collateral, using the same matrix $Y^{g(j)}$,
} 
Theorem 2 Let $J^{\star}$ be the set of promises not satisfying the assumptions of Theorem 1. Equilibrium exists:

(i) for exogenous collateral coefficients if every promise $j \in J^{*}$ is a nominal promise backed by a real collateral instrument,

(ii) for exogenous collateral coefficients if every promise $j \in J^{*}$ is a real promise backed by a nominal collateral instrument,

(iii) for endogenously determined margin requirements, if every $j \in J^{*}$ is not a real promise backed by real collateral.

(See the appendix for a proof).

Intuitively, in cases (i) or (ii), we do not have the homogeneity of real allocations with respect to a generalized scaling up of prices at all immediately following nodes. Hence, the sum of marginal penalty effects, across these nodes, gets dominated by the sum of marginal income effects across these nodes, in case (i) for high inflation rates $\sigma_{\eta}$ at all these nodes and in case (ii) for low inflation rates $\sigma_{\eta}$ at all these nodes. But when the nominal promise/real collateral, real promise/nominal collateral or nominal promise/nominal collateral contracts coexist, there are trade-offs on the inflation rates, which can be overcome when margin requirements are endogenously determined.

Notice that for a contract with a nominal promise $j$ backed by real collateral, (11) becomes:

$$
\sum_{\sigma \in \xi^{+}} \sigma_{\eta}^{-1} \max \left\{\lambda_{j}^{i}(\eta), \mu^{i}(\eta)\right\} b_{\eta}^{j} \leq \sum_{\sigma \in \xi^{+}} \min \left\{\lambda_{j}^{i}(\eta), \mu^{i}(\eta)\right\} p(\eta) Y(\eta) C^{j}(\eta),
$$

whereas for a contract with a real promise $j$ backed by nominal collateral, (11) becomes:

$$
\sum_{\sigma \in \xi^{+}} \max \left\{\lambda_{j}^{i}(\eta), \mu^{i}(\eta)\right\} p(\eta) A^{j}(\eta) \leq \sum_{\sigma \in \xi^{+}} \sigma_{\eta}^{-1} \min \left\{\lambda_{j}^{i}(\eta), \mu^{i}(\eta)\right\} \tilde{y}_{\eta}^{j} C^{j}(\xi)
$$

and, finally, for a nominal promise backed by a nominal collateral, (11) is:

$$
\sum_{\sigma \in \xi^{+}} \sigma_{\eta}^{-1} \max \left\{\lambda_{j}^{i}(\eta), \mu^{i}(\eta)\right\} b_{\eta}^{j} \leq \sum_{\sigma \in \xi^{+}} \sigma_{\eta}^{-1} \min \left\{\lambda_{j}^{i}(\eta), \mu^{i}(\eta)\right\} \tilde{y}_{\eta}^{j} C^{j}(\xi)
$$

Theorem 2 allowed for direct utility gains from collateral in the case of nominal promises backed by real collateral, by showing that (13) holds for every agent. If (13) held for just one agent and there were no utility gains from collateral, then condition (8) in the Proposition would still be verified and there would exist an equilibrium for the infinite horizon economy.

\section{Example 2}

For the economy of Example 1, take agent (1) and the pair of contracts: one nominal-real with $b_{u_{\xi}}^{1}=1, \quad b_{d_{\xi}}^{1}=2$ and $Y_{u_{\xi}}=(1,0), Y_{d_{\xi}}=(1,0), C^{1}=(0,1)$ and another nominal-nominal with $b_{u_{\xi}}^{2}=1, b_{d_{\xi}}^{2}=3$ and $\tilde{y}_{u_{\xi}}=\tilde{y}_{d_{\xi}}=1, C^{2}$ to be determined. For $\lambda_{j}^{(1)}(\eta)=\beta^{t(\eta)}\left(\frac{1}{2}\right)^{t(\eta)} \delta_{\eta}^{(1) j}$, let $\delta_{u_{\xi}}^{(1) j}=2$ and $\delta_{\eta}^{(1) j}=1$ otherwise $(j=1,2)$. Then, conditions (13) and (15) hold (for $j=1$ and $j=2$ respectively), with an exogenous collateral requirement for $j=1$ and endogenous ones for $j=2$.

These conditions are:

$$
2 \sigma_{u_{\xi}}^{-1} b_{u_{\xi}}^{1}+\sigma_{d_{\xi}}^{-1} b_{d_{\xi}}^{1} \leq Y_{u_{\xi}}+Y_{d_{\xi}}
$$

we see that as $\left(\sigma_{\eta}\right)_{\eta \in \xi^{+}}$is multiplied by $\alpha>0$, we can preserve both the bundle and the portfolio, at the same relative spot prices ans asset prices, by adjusting $C_{g(j)}^{j}$ (multiplying by $\alpha>0$ in this case), so the same quotient set still works. 


$$
2 \sigma_{u_{\xi}}^{-1} b_{u_{\xi}}^{2}+\sigma_{d_{\xi}}^{-1} b_{d_{\xi}}^{2} \leq\left(\sigma_{u_{\xi}}^{-1} \tilde{y}_{u_{\xi}}+\sigma_{d_{\xi}}^{-1} \tilde{y}_{d_{\xi}}\right) C^{2} .
$$

Holding as equalities for $\sigma_{u_{\xi}}^{-1}=\sigma_{d_{\xi}}^{-1}=0.5$ and $C^{2}=2.5$, implying that at $u_{\xi}$, both promises are above collateral values (with opportunity for default, which will not be used as $\lambda_{u_{\xi}}^{(1) j}>\mu_{u_{\xi}}^{(1)}$ ) while at $d_{\xi}$ the first promise matches the collateral values whereas the second one falls below it.

If we had tried to endogeneize collateral coefficients (by scaling them up or down) in the case of real promises backed by real collateral, with the purpose of having (11) verified in equilibrium, we would be left with a condition that might not be far from requiring collateral to be so high that the promises always fall below it. The condition on the scale factor $\alpha_{\xi}^{j}$ would be:

$$
\alpha_{\xi}^{j} \sum_{\sigma \in \xi^{+}} \min \left\{\lambda_{j}^{i}(\eta), \mu^{i}(\eta)\right\} p(\eta) Y^{j}(\eta) C^{j}(\eta) \geq \sum_{\sigma \in \xi^{+}} \max \left\{\lambda_{j}^{i}(\eta), \mu^{i}(\eta)\right\} p(\eta) A^{j}(\eta) .
$$

This is a much more stringent condition than the ones used in the proof of Theorem 2 (on $\alpha_{\xi}^{j}, j \in J_{1}$, or on $\gamma_{\xi}^{j}, j \in \tilde{J}$, see proof), which could be combined with the flexibility that the choice of inflation ratios gave us in the case of contracts with nominal promises or nominal collateral.

\section{Should The EQUiLIBRIUM BE REFined?}

\subsection{Do trivial equilibria exist?}

When promises are not collateralized, a trivial no-trade equilibrium can be found by setting $K^{j}(\xi)=0$, as already remarked by Dubey, Geanakoplos and Shubik (2005). In fact, if for each node $\xi$ and for each promise $j, C^{j}(\xi)=0$, let $\theta_{j}^{i}(\xi)=\varphi_{j}^{i}(\xi)=0, \forall(i, j, \xi)$ and (6) holds. Now, (5) can be rewritten as follows:

$$
q^{j}(\xi) \leq \sum_{\eta \in \xi^{+}} \min \left\{\mu^{i}(\eta), \tilde{\lambda}^{i}(\eta)\right\} \frac{1}{\mu^{i}(\xi)} p(\eta) A^{j}(\eta) \equiv \Psi_{j}^{i}(\xi),
$$

and let $q^{j}(\xi)=\min _{i} \Psi^{i}(\xi)$. Finally, (4) is replaced by $\mu^{i}(\xi) \geq \tilde{\lambda}_{j}^{i}(\xi) d_{j}^{i}(\xi)$ and $\left(\mu^{i}(\xi)-\tilde{\lambda}_{j}^{i}(\xi) d_{j}^{i}(\xi)\right) \Delta_{j}^{i}(\xi)=$ 0 , so $d_{j}^{i}(\xi)$ is chosen less than one when $\mu^{i}(\xi)<\tilde{\lambda}_{j}^{i}(\xi)$.

However, when promises are collateralized, $K^{j}(\eta)$ is bounded from below by $\frac{\operatorname{Min}_{j}(\eta)}{p(\eta) A^{j}(\eta)}$ when $p(\eta) A^{j}(\eta)>$ 0 . Does a trivial equilibrium exist by setting $K^{j}(\eta)$ equal to this lower bound and promise positions equal to zero? Condition (6) holds for all $i$ by making $q^{j}(\xi)=\max _{i} \sum_{\eta \in \xi^{+}} \frac{\mu^{i}(\eta)}{\mu^{i}(\xi)} \operatorname{MIN}_{j}(\eta)$. But, condition (5) requires:

$$
\begin{aligned}
& q^{j}(\xi)-\sum_{\eta \in \xi^{+}} \frac{\mu^{i}(\eta)}{\mu^{i}(\xi)} \operatorname{MIN}_{j}(\eta) \\
\leq & \sum_{\eta \in \xi^{+}} \frac{\tilde{\lambda}_{j}^{i}(\eta) d_{j}^{i}(\eta)}{\mu^{i}(\xi)}\left(p(\eta) A^{j}(\eta)-\operatorname{MIN}_{j}(\eta)\right)+f_{j}^{i}(\xi),
\end{aligned}
$$

where $f_{j}^{i}(\xi) \equiv p(\xi) C^{j}(\xi)-\frac{v_{\xi}^{\prime} \cdot C^{j}(\xi)}{\mu^{i}(\xi)}-\sum_{\eta \in \xi^{+}} \frac{\mu^{i}(\eta)}{\mu^{i}(\xi)} p(\eta) Y(\eta) C^{j}(\xi) \geq 0\left(\right.$ and $f_{j}^{i}(\xi)=0$ when $x^{i}(\xi, g)>0$ for $\left.g: C_{g}^{j}(\xi)>0\right)$.

Clearly, for the agent $i$ with the highest $\sum_{\eta \in \xi^{+}} \frac{\mu^{i}(\eta)}{\mu^{i}(\xi)} \operatorname{MIN}_{j}(\xi)$, this inequality holds, as the left hand side is zero. But, for other agents, there is no reason why the gap on the left hand side can be covered by the terms on the right hand side (choosing $d_{j}^{i}(\eta)<1$ would only hurt and if $f^{i}(\xi)=0$ the difficulty is even worse). 
If markets were complete, that is, when $\frac{\mu^{i}(\eta)}{\mu^{i}(\xi)}$ were common across agents, a tradeless equilibrium could be trivially found.

Hence, when $\# \xi^{+}>\# J(\xi)$, no-trade equilibrium cannot be trivially found by setting $K^{j}(\xi)=$ $\frac{\operatorname{MIN}_{j}(\xi)}{p(\xi) A^{j}(\xi)}$, but such equilibrium certainly exists if markets become endogenously complete.

\subsection{Removing unduly expectations.}

Although it is not necessary to refine the equilibrium concept to rule out trivial equilibria, we may want to do it always to avoid unduly expectations about delivery rates. Recall that in the absence of collateral, Dubey, Geanakoplos and Shubik (2005) proposed a refinement consisting of focusing on equilibria which were limits (as $\varepsilon \longrightarrow 0$ ) of equilibria of economies with an artificial agent buying and selling $\varepsilon$ units of each asset, delivering fully but receiving $K^{j}(\xi) p(\xi) A^{j}(\xi)$. Commodity market clearing was adjusted to accommodate the fact that this agent was injecting $\sum_{j} \varepsilon\left(1-K^{j}(\xi)\right) A^{j}(\xi)$ goods in the economy. The delivery rate $K^{j}(\xi)$ was such that $K^{j}(\xi) p(\xi) A^{j}(\xi)\left(\varepsilon+\sum_{i} \theta_{j}^{i}(\xi)\right)=\varepsilon p(\xi) A^{j}(\xi)+\sum_{i} \Delta_{j}^{i}(\xi)$. This refinement not just eliminated the above trivial equilibria but also got rid of over-pessimistic expectations. Without it, when all agent are "strictly conscientious" (that is, $\tilde{\lambda}_{j}^{i}(\xi)>\mu^{i}(\xi)$ ), we would have the pathological result that $K^{j}(\xi)$ could be set equal to zero in the trivial no-trade equilibrium.

We may want to refine the equilibrium to avoid irrational over-pessimistic expectations, and why not, also over-optimistic expectations. The above refinement eliminates pathological equilibria with $K^{j}(\xi)=\frac{\operatorname{MIN}_{j}(\xi)}{p(\xi) A^{j}(\xi)}$, even though $\tilde{\lambda}_{j}^{i}(\xi)>\mu^{i}(\xi), \forall i$ (as in the example they discuss). However, this refinement seems to be too strong, as it eliminates also equilibria where some promise $j$ is not traded but $\tilde{\lambda}_{j}^{i}(\xi)<\mu^{i}(\xi), \forall i$, and $K^{j}(\xi)=\frac{\operatorname{MIN}_{j}(\xi)}{p(\xi) A^{j}(\xi)}$ (as expected) if one cannot show that along the sequence of equilibria for the $\varepsilon$-economy the true agents are trading promise $j$, even if some agents are actually on the margin of doing it (with conditions (5) and (6) holding as equalities).

Moreover, in infinite horizon economies, this shortcoming of the above refinement becomes a serious problem. In fact, unduly high expectations about $K^{j}(\eta)$ make asset prices of non-traded promises become over estimated due to (6) and Ponzi schemes may occur spuriously (as $p(\xi) C^{j}(\xi)-q^{j}(\xi)$ may be fictitiously negative).

Actually, when avoiding unduly ((pessimistic and optimistic) expectations, what is important is to eliminate delivery beliefs that are inconsistent with the penalty functions of agents who are on the margin of selling the promise. As Dubey, Geanakoplos and Shubik (2005) stressed, these on-the-verge agents should pin down what the delivery rates are.

\subsection{A different refinement concept.}

We propose a different refinement. Let $E=(\bar{p}, \bar{q}, \bar{K}, \bar{x}, \bar{\theta}, \bar{\varphi}, \bar{\Delta})$ be an equilibrium and $\tilde{J}_{\xi}(E)$ be the set of promises that are not traded at node $\xi$. The delivery rate of $j \in \tilde{J}_{\xi}(E)$ at each node $\eta \in \xi^{+}$ should be consistent with the penalty coefficients and marginal utilities of income of agents that might be already purchasing what can serve as collateral for $j$ and are also on-the-verge of selling $j$.

Before defining the refinement more precisely, we should be explicit about what we mean by on-theverge of selling promise $j$ at node $\xi$. By this we mean that (5) should hold as an equality for $\left(d_{j}^{i}(\eta)\right)_{\eta \in \xi^{+}}$ consistent with positive sales. If $j \in \tilde{J}_{\xi}(E)$ we just know that (5) holds with $\left(d_{j}^{i}(\eta)\right)_{\eta \in \xi^{+}}$consistent with null sales. However, if $\tilde{\lambda}_{j}^{i}(\eta)<\mu^{i}(\eta)$ and $j \in \tilde{J}_{\xi}(E)$, (5) might hold as an equality for $d_{j}^{i}(\eta)<1$ (which is a supergradient of the map $y \longmapsto \max \{0, y\}$ at 0$)$, but for $i$ to be on-the-verge of selling, such $d_{j}^{i}(\eta)$ does not work. We would like to have the Kuhn-Tucker conditions of $i$ ready for him to become a seller 
(and, therefore, a deliver with maximal default, so with $d_{j}^{i}(\eta)=1$ for $\eta: p(\eta) A^{j}(\eta)>\operatorname{MIN}_{j}(\eta)$ ). For $\tilde{\lambda}_{j}^{i}(\eta)>\mu^{i}(\eta)$, this problem does not occur as $d_{j}^{i}(\eta)$ must be less than 1 (by (4)), which is compatible with both a null sale or a sale without default. For $\tilde{\lambda}_{j}^{i}(\eta)=\mu^{i}(\eta)$, the problem is also absent as (i) if $d_{j}^{i}(\eta)=1$ and $\rho_{j}^{i}(\eta)=0$, we can have either a null sale or a sale (with default or not) or (ii) if $d_{j}^{i}(\eta)<1$, we can have either a null sale or a sale without default.

That is, an agent on-the-verge of selling promise $j$ at node $\xi$ should be ready to deliver at the next nodes $\eta \in \xi^{+}$according to the optimality criterion (4) (for the right supergradient $d_{j}^{i}(\eta)$ ), given his penalty coefficients $\tilde{\lambda}_{j}^{i}(\eta)$ and the marginal utilities of income $\mu^{i}(\eta)$.

Let us assume that different promises use different collateral instruments and that each promise uses just one collateral instrument.

Assumption [C]. The mapping $j \longmapsto\left\{g \in G: C_{g}^{j}(\xi)>0\right\}$ is an injective function that does not change from node to node. Denote by $g(j)$ the element $g \in G$ such that $C_{g}^{j}(\xi)>0$.

The node-invariance was assumed to simplify the notations. Now, for each node $\xi$ and each promise $j$, let $N_{\xi}^{j}(E)=\left\{i: \tilde{x}^{i}(\xi, g(j))>0\right.$ at E $\}$ and let $V_{\xi}^{j}(E)=\left\{i \in N_{\xi}^{j}(E):(5)\right.$ holds with equality for $i$ at $\mathrm{E}$ with $d_{j}^{i}(\eta) \in[0,1]$ satisfying $(4)$ and such that $d_{j}^{i}(\eta)=1$ if $\left.\tilde{\lambda}_{j}^{i}(\eta)<\mu^{i}(\eta), \eta \in \xi^{+}\right\}$.

Notice that if promise $j$ is traded at $\xi$, then $V_{\xi}^{j}(E) \neq \emptyset$. When this set is nonempty although $j$ is not traded at $\xi$, we should check whether the delivery expectations are consistent with the relation between penalties and marginal utilities for income of agents in this set.

Definition 5.1 Given an equilibrium $E=(\bar{p}, \bar{q}, \bar{K}, \bar{x}, \bar{\theta}, \bar{\varphi}, \bar{\Delta})$, let an auxiliary $\epsilon$-economy differ from the original economy by adding another agent, called "the government", that collects at node $\xi$ lumpsum taxes $t_{j}^{i}(\xi)$ from consumers in $V_{\xi}^{j}(E), j \in \tilde{J}_{\xi}(E)$, with $\sum_{i \in V_{\xi}^{j}(E)} t_{j}^{i}(\xi)=\bar{q}^{j}(\xi) \varepsilon$, spends the tax revenue purchasing $\theta_{j}^{G}(\xi)$ units of promise $j$ at $\xi$, and, then, at $\xi \in \xi^{+}$, gives lump-sum subsidies $s_{j}^{i}(\eta)$ to consumers in $V_{\xi}^{j}(E)$ using returns from the purchase done at $\xi$. In an equilibrium for the $\varepsilon-$ economy the government choice variables should satisfy $K^{j}(\eta) p(\eta) A^{j}(\eta) \theta_{j}^{G}(\xi)=\sum_{i \in V_{\xi}^{j}(E)} s_{j}^{i}(\eta)$. At the same time, the delivery rate should be such that $K^{j}(\eta) p(\eta) A^{j}(\eta)\left(\theta_{j}^{G}(\xi)+\sum_{i} \theta_{j}^{i}(\xi)\right)=\sum_{i} \Delta_{j}^{i}(\eta)$. Market clearing for $j$ requires now $\sum_{i} \varphi_{j}^{i}(\xi)=\theta_{j}^{G}(\xi)+\sum_{i} \theta_{j}^{i}(\xi)$.

We will be interested in a special class of equilibria for the $\varepsilon$-economy, called $E_{\varepsilon}$ equilibria, where only the government purchases promise $j \in \tilde{J}_{\xi}(E)$, only consumers in $V_{\xi}^{j}(E)$ sell it and marginal utilities of income $\mu^{i}(\eta), \eta \in \xi^{+}$, for $i \in V_{\xi}^{j}(E)$, are as in the original equilibrium $E$, so that agents $V_{\xi}^{j}(E)$ are just as willing to default as they were at the original equilibrium $E$. Let $\gamma_{j}^{i}(\eta) \in[0,1]$ be such that $\gamma_{j}^{i}(\eta)=1$ if $\tilde{\lambda}_{j}^{i}(\eta)>\mu^{i}(\eta)$ and $\gamma_{j}^{i}(\eta)=0$ if $\tilde{\lambda}_{j}^{i}(\eta)<\mu^{i}(\eta)$. In such equilibria, $t_{j}^{i}(\xi)=\frac{\bar{q}^{j}(\xi) \varepsilon}{\# V_{\xi}^{j}(E)}$, $s_{j}^{i}(\eta)=\left[\gamma_{j}^{i}(\eta) p(\eta) A^{j}(\eta)+\left(1-\gamma_{j}^{i}(\eta)\right) \operatorname{MIN}_{j}(\eta)\right] \frac{\varepsilon}{\# V_{\xi}^{j}(E)}$ and $K^{j}(\eta) p(\eta) A^{j}(\eta)=\frac{1}{\varepsilon} \sum_{i \in V_{\xi}^{j}(E)} s_{j}^{i}(\xi)$, whenever $\bar{q}^{j}(\xi)>0$

Definition 5.2 An equilibrium $E=(\bar{p}, \bar{q}, \bar{K}, \bar{x}, \bar{\theta}, \bar{\varphi}, \bar{\Delta})$ is a refined equilibrium if, whenever $V_{\xi}^{j}(E) \neq$ $\emptyset$, for some $j \in \tilde{J}_{\xi}(E)$ and some $\xi, E$ is a limit (in the product topology of the countable tree) of a sequence of equilibria $E_{\varepsilon}$ for $\varepsilon_{n}$-economy (as $\left.\varepsilon_{n} \longrightarrow 0\right)$.

Clearly, if at the original equilibrium $E, \bar{q}^{j}(\xi)>0$, and, for every $\eta \in \xi^{+}$such that $p(\eta) A^{j}(\eta)>$ $0, \bar{K}^{j}(\eta)$ is weighted average with weights $\beta_{j}^{i}(\xi)$, of the individual delivery rates $\zeta_{j}^{i}(\eta) \equiv\left[\gamma_{j}^{i}(\eta) p(\eta) A^{j}(\eta)+\right.$ $\left.\left(1-\gamma_{j}^{i}(\eta)\right) \operatorname{MIN}_{j}(\eta)\right] / p(\eta) A^{j}(\eta)$ of agents in $V_{\xi}^{j}(E)$ (possibly with some null weights) for every $j$ in $\tilde{J}_{\xi}(E)$, 
as it is known to be the case for traded promises $j \notin \tilde{J}_{\xi}(E)$, then for each $\varepsilon$, we have an equilibrium $E_{\varepsilon}$ that differs from $E$ only by making, for $i \in V_{\xi}^{j}(E), \varphi_{j}^{i}(\xi)=\beta_{j}^{i}(\xi) \varepsilon, t_{j}^{i}(\xi)=\beta_{j}^{i}(\xi) \varepsilon \bar{q}^{j}(\xi)$ and $x^{i}(\xi, g(j))=\bar{x}^{i}(\xi, g(j))-C_{g(j)}^{j} \beta_{j}^{i}(\xi) \varepsilon$. Then, $E$ is a refined equilibrium.

\subsection{Properties of the refinement.}

What does the above refinement do when agents are strictly conscientious?

Claim 5.1 If $E$ is such that $V_{\xi}^{j}(E) \neq \emptyset$ and $\tilde{\lambda}_{j}^{i}(\eta)>\mu^{i}(\eta), \eta \in \xi^{+}, \forall i \in V_{\xi}^{j}(E)$, then $E$ is a refined equilibrium if and only if $K^{j}(\eta)=1$ for $\eta: p(\eta) A^{j}(\eta)>0$.

Proof. (if) For each $\varepsilon>0$, there exists an $E_{\epsilon}$-equilibrium with $K_{\varepsilon}^{j}(\eta)=\frac{\sum_{i \in V_{\xi}^{j}(E)} \gamma_{j}^{i}(\eta)}{\# V_{\xi}^{j}(E)}=1$ (as $\left.\gamma_{j}^{i}(\eta)=1, \forall i \in V_{\xi}^{j}(E)\right)$, for $\eta: p(\eta) A^{j}(\eta)>0$.

(Only if) For each $\varepsilon>0$, at any $E_{\epsilon}$ the $\varepsilon$-economy we must have $\Delta_{j}^{i}(\eta)=p(\eta) A^{j}(\eta) \varphi_{j}^{i}(\xi)$, so $K^{j}(\eta)=1$, for $\eta: p(\eta) A^{j}(\eta)>0$.

To see that the proposed refinement manages also (as the one proposed by Dubey, Geanakoplos and Shubik (2005)) to eliminate spurious equilibria with unduly pessimist expectations about the delivery of strictly conscientious agents agents, we combine the above claim with the next one:

Claim 5.2 If $E$ is such that for $j \in \tilde{J}_{\xi}(E), \tilde{\lambda}_{j}^{i}(\eta)>\mu^{i}(\eta), \forall i$, then $V_{\xi}^{j}(E) \neq \emptyset$.

Proof. In fact, under Assumption [C], if $j \in \tilde{J}_{\xi}(E)$, there exists some agent $i$ such that $x^{i}(\xi, g(j))>0$. For this agent (as $(7)$ holds with equality for $g(j)$ at $\xi$ ), condition (5) becomes:

$$
\sum_{\eta \in \xi^{+}} \tilde{\lambda}_{j}^{i}(\eta) d_{j}^{i}(\eta)\left(p(\eta) A^{j}(\eta)-\operatorname{MIN}_{j}(\eta)\right) \geq \mu^{i}(\xi) q^{j}(\xi)-\sum_{\eta \in \xi^{+}} \mu^{i}(\eta) K^{j}(\eta) p(\eta) A^{j}(\eta),
$$

where the right hand side is non-negative, by (6). Now, $\tilde{\lambda}_{j}^{i}(\eta)>\mu^{i}(\eta)$ implies, by $(4)$, that $d_{j}^{i}(\eta)$ can be chosen in $[0,1)$. By choosing $d_{j}^{i}(\eta)$ small enough (for $\eta \in \xi^{+}$such that $p(\eta) A^{j}(\eta)>\operatorname{MIN}_{j}(\eta)$ ) we get the equality in (16), as desired.

Let us see what can be said about the refinement of equilibria found in Theorem 1.

Claim 5.3 Under the assumptions of Theorem 1 and Assumption [C], there exists a refined equilibrium, where $K^{j}(\xi) p(\xi) A^{j}(\xi)=\operatorname{MIN}_{j}(\xi), \forall j, \forall \xi$.

Proof. In fact, at the equilibrium $E$ found in Theorem 1 , we had $K(\xi) p(\xi) A^{j}(\xi)=\operatorname{MIN}_{j}(\xi), \forall j, \xi$. If $V_{\xi}^{j}(E) \neq \emptyset$, for $j \in \tilde{J}_{\xi}(E)$, then at the $E_{\varepsilon}$ equilibrium for each $\varepsilon$-economy, we have $p(\eta) A^{j}(\eta) \hat{K}_{\varepsilon}^{j}(\eta)=$ $\frac{\sum_{i \in V_{\xi}^{j}(E)}\left(1-\gamma_{j}^{i}(\eta)\right) \operatorname{MIN}_{j}(\eta)}{\# V_{\xi}^{j}(E)}, \forall \eta \in \xi^{+}$, where $\gamma_{j}^{i}(\eta)=0, \forall i \in V_{\xi}^{j}(E)$. So, $K^{j}(\eta)=\hat{K}_{\varepsilon}^{j}(\eta), \forall \eta \in \xi^{+}$. If $V_{\xi}^{j}(E)=\emptyset$, the result is immediate.

Are there refined equilibria under the assumptions of Theorem 2? First, we will show that, for finite horizon economies, we can always find a refined equilibrium (as long as Assumption [C] is satisfied). Secondly, under the assumptions of Theorem 2, these refined equilibria of finite finite horizon economies induce a refined equilibrium for the infinite horizon economy. 
Claim 5.4 Under Assumption [C], in a finite horizon economy, there exists a refined equilibrium, where $K^{j}(\xi)$ belongs to the convex hull of $\left(\zeta_{j}^{i}(\xi)\right)_{i \in V_{\xi}^{j}(E)}$, if $p(\xi) A^{j}(\xi)>0$ and $V_{\xi}^{j}(E) \neq \emptyset$, for $j \in \tilde{J}_{\xi}(E)$.

Proof. See Appendix.

Claim 5.5 Under the assumptions of Theorem 2 and Assumption [C], there exists a refined equilibrium for the infinite horizon economy.

Proof. By the previous claim, finite horizon economies have refined equilibria. Actually, under the assumptions of Theorem 2, these refined equilibria of finite horizon economies satisfy (11), (12) or (13). Let the horizon $T$ go to $\infty$ : the cluster point of the finite horizon equilibria satisfies (8) and is therefore an equilibrium for the infinite horizon economy. Moreover, it is refined equilibrium as, when $p(\eta) A^{j}(\eta)>0, K^{j}(\eta)=\sum_{i \in V_{\xi}^{j}(E)} \beta_{j}^{i}(\xi) \zeta_{j}^{i}(\eta), \forall \eta \in \xi^{+},\left(\beta_{j}^{i}(\xi)\right)_{i} \in \Delta^{\# V_{\xi}^{j}(E)-1}$, whenever $V_{\xi}^{j}(E) \neq \emptyset$, for $j \in \tilde{J}_{\xi}(E)$ (since this relation held at the refined equilibria of finite horizon economies found in the previous claim).

\section{Appendix}

For each $\mu$ and for each agent $i$, Let us define the Lagrangian function associated with agent $i^{\prime}$ problem as follows:

$$
\begin{aligned}
L_{\xi}^{i}\left(Z_{\xi}, Z_{\xi^{-}}, \mu, p, q, K\right) & =v_{\xi}^{i}\left(Z_{\xi}\right)-\sum_{j} \tilde{\lambda}_{j}^{i}(\xi)\left[p(\xi) A^{j}(\xi) \varphi_{j}^{i}\left(\xi^{-}\right)-\Delta_{j}^{i}(\xi)\right]^{+} \\
& -\mu(\xi)\left[p(\xi) \cdot\left(x^{i}(\xi)+C(\xi) \varphi^{i}(\xi)\right)+q(\xi) \cdot\left(\theta^{i}(\xi)-\varphi^{i}(\xi)\right)\right. \\
& -p(\xi) \omega^{i}(\xi)-p(\xi) Y(\xi)\left(x^{i}\left(\xi^{-}\right)-C\left(\xi^{-}\right) \varphi^{i}\left(\xi^{-}\right)\right)+\sum_{j \in J\left(\xi^{-}\right)} \Delta_{j}^{i}(\xi) \\
& \left.-\sum_{j \in J\left(\xi^{-}\right)} K^{j}(\xi) p(\xi) A^{j}(\xi) \theta_{j}^{i}\left(\xi^{-}\right)\right] \\
& -\sum_{j \in J\left(\xi^{-}\right)} \rho^{j}(\xi)\left[\operatorname{MIN}_{j}(\xi) \varphi_{j}^{i}\left(\xi^{-}\right)-\Delta_{j}^{i}(\xi)\right]
\end{aligned}
$$

Lemma 1 For each node $\xi \in D$ and for all economies with finite horizon $T \geq t(\xi)$, one has:

$$
0 \leq \mu^{i}(\xi)<\frac{U^{i}(\mathcal{W})}{\underline{W^{i}}(\xi)\left\|\bar{p}^{T}(\xi)\right\|_{1}},
$$

where $\mathcal{W}^{i}(\xi)=\sum_{\eta \leq \xi} \prod_{s=\eta}^{\xi} Y(s) \omega^{i}(\eta)$ and $\underline{W^{i}}(\xi)=\min _{g} \mathcal{W}^{i}(\xi, g)$.

Proof of Lemma 1. For $t \leq T$, let $Z=(Z(\xi))_{\xi \in D^{T}}$ be such that $Z(\xi)=\left(\mathcal{W}^{i}(\xi), 0,0,0\right)$ if $\xi \in D^{t-1}$ and $Z(\xi)=0$ otherwise. Now,

$$
\sum_{\xi \in D^{T}} \mathcal{L}_{\xi}^{i}\left(Z^{i T}(\xi), Z^{i T}\left(\xi^{-}\right), \mu^{i T}(\xi), \bar{p}^{T}, \bar{q}^{T}\right) \leq \sum_{\xi \in D^{T}} v_{\xi}^{i}\left(\bar{Z}^{i T}(\xi)\right)
$$

It then follows that $\sum_{\xi \in D_{t}} \mu^{i}(\xi) p(\xi) \mathcal{W}^{i}(\xi) \leq \sum_{\xi \in D^{t}} v_{\xi}^{i}\left(\bar{Z}^{i T}(\xi)\right)$. The lemma follows then as $v_{\xi}^{i}\left(\bar{z}^{i T}(\xi)\right) \leq$ $v_{\xi}^{i}\left(\mathcal{W}_{\xi}\right)$, where $\mathcal{W}_{\xi}:=\sum_{i \in I} \mathcal{W}_{\xi}^{i}$ 
Lemma 2 Let $\mathcal{W}_{\xi}=\sum_{i} \mathcal{W}^{i}(\xi)$. Then, $\delta^{+} v_{\xi}^{i}\left(x^{i}(\xi), \kappa_{\xi}\right)$ has a positive minimum on the set of bundles $z \leq \mathcal{W}_{\xi}$, where $\kappa_{\xi}=\mathbb{I}$ or $\kappa_{\xi}=b(\xi)$.

Proof of Lemma 2. Denote by $A:=B\left(0, \mathcal{W}_{\xi}\right) \cap \mathbb{R}_{+}^{G}+\varepsilon \kappa_{\xi}$, where $B(0, \alpha)$ denotes the ball with center 0 and radius $\alpha$. Now, $\delta^{+} v_{\xi}^{i}\left(x^{i}(\xi), \kappa_{\xi}\right) \geq \min _{z \in A} \delta^{+} v_{\xi}^{i}\left(z, \kappa_{\xi}\right):=r_{\xi}^{i}\left(\kappa_{\xi}\right)$. In fact, let $\tau \in \mathbb{R}_{+}^{G}$ and $S_{\tau}=\left\{z \in \mathbb{R}^{G}: z=a \kappa_{\xi}+\tau\right.$, for some $\left.a\right\}$, then for any $z \in S_{\tau} \cap \operatorname{dom} \partial v_{\xi}^{i}, \delta^{+} v_{\sigma}^{i}\left(z, \kappa_{\sigma}\right) \geq \delta^{+} v_{\sigma}^{i}\left(\bar{z}, \kappa_{\sigma}\right)$, where $\{\bar{z}\}=S_{\tau} \cap A$ (by monotonicity of this directional derivative on the straight line $S_{\tau}$ ). The minimum of $\delta^{+} v_{\sigma}^{i}\left(z, \kappa_{\sigma}\right)$ over $z \in \operatorname{dom} \partial v_{\sigma}^{i}$ such that $z \leq \mathcal{W}_{\xi}$ is therefore attained on the compact set $A$.

Lemma 3 The sum of spot prices is bounded away from zero, at each node (uniformly bounded in the finite horizon $T$ and, therefore, also bounded in the infinite horizon economy).

Proof of Lemma 3. This can be established as in (b.2) of Lemma A.2 in Páscoa and Seghir (2009) using an upper bound $m_{g}^{j}(\xi)$ on $\frac{q^{j}(\xi)}{\sum_{g} p(\xi, g)}$. Under assumptions [E] and [U], we get $m_{j}(\xi)=\bar{C}^{j}(\xi)+$ $\frac{1}{r_{\xi}^{i}(\mathbb{I})} \sum_{\eta \in \xi^{+}} \frac{\tilde{\lambda}_{j}(\eta) \bar{A}^{j}(\eta)}{\underline{b}(\eta)}$, where $r_{\xi}^{i}(\mathbb{I})$ is the minimum of the right derivative of $v_{\xi}^{i}$ in the direction of $\mathbb{I}$, over all feasible bundles (see Lemma 2 above) ${ }^{7}$.

Remark A.1 It follows by Lemma 1 and Lemma 3 that multipliers $\mu^{i}(\xi)$ have upper bounds that are independent of prices and of the terminal horizon $T$ of the economy, as $\underline{W}^{i}(\xi)>0$ by Assumption [E]. Moreover, it follows from equation (4) that $\rho^{i}(\xi)$ also has an upper bound independent of prices and $T$.

Proof of Proposition. Let the vectors $L_{1 \xi}^{i}$ and $L_{2 \xi}^{i}$ be partial super-gradients of $L^{i}(Z)$ with respect to the current and past decision variables, respectively, verifying the Kuhn-Tucker conditions at the cluster point of finite horizon equilibria. These conditions ((4) through (7), holding as equalities when the respective variables are positive) can be written as:

$$
\begin{gathered}
L_{1 \xi}^{i}\left(\bar{Z}^{i}\right)+\sum_{\eta \in \xi^{+}} L_{2 \eta}^{i}\left(\bar{Z}^{i}\right) \leq 0 \\
\left(L_{1 \xi}^{i}\left(\bar{Z}^{i}\right)+\sum_{\eta \in \xi^{+}} L_{2 \eta}^{i}\left(\bar{Z}^{i}\right)\right) \bar{Z}_{\xi}^{i}=0 .
\end{gathered}
$$

Then, one has:

$$
\begin{aligned}
\Pi^{i T}(Z)-\Pi^{i T}\left(\bar{Z}^{i}\right) \leq & \sum_{\xi: t(\xi) \leq T}\left(L_{\xi}^{i}(Z)-L_{\xi}^{i}\left(\bar{Z}^{i}\right)\right) \\
\leq & \sum_{\xi: t(\xi) \leq T}\left(L_{1 \xi}^{i}\left(\bar{Z}^{i}\right), L_{2 \xi}^{i}\left(\bar{Z}^{i}\right)\right)\left(Z_{\xi}-\bar{Z}^{i}\right) \\
= & \sum_{\xi: t(\xi)<T}\left(L_{1 \xi}^{i}\left(\bar{Z}^{i}\right)+\sum_{\eta \in \xi^{+}} L_{2 \eta}^{i}\left(\bar{Z}^{i}\right)\right) Z_{\xi}+\sum_{\xi: t(\xi)=T} L_{1 \xi}^{i}\left(\bar{Z}^{i}\right) Z_{\xi} \\
& -\sum_{\xi: t(\xi)<T}\left(L_{1 \xi}^{i}\left(\bar{Z}^{i}\right)+\sum_{\eta \in \xi^{+}} L_{2 \eta}^{i}\left(\bar{Z}^{i}\right)\right) \bar{Z}_{\xi}^{i}-\sum_{\xi: t(\xi)=T} L_{1 \xi}^{i}\left(\bar{Z}^{i}\right) \bar{Z}_{\xi}^{i} .
\end{aligned}
$$

${ }^{7}$ The upper bound $\sum_{g}\left(v_{\sigma, g}^{i}\right)_{+}^{\prime}\left(\frac{W I}{1-k}\right)$ for the relative price $\frac{q^{j}(\xi)}{\sum_{g} p(\xi, g)}$, given in item (b.1) of Lemma A.2 in Páscoa and Seghir (2009), while correct for utilities that are separable in commodities, should, in general, be replaced by the upper bound given now by Lemma 3 . 
Now, $\sum_{\xi: t(\xi)=T} \sum_{\eta \in \xi^{+}} L_{2 \eta}^{i}\left(\bar{Z}^{i}\right) \bar{Z}_{\xi}^{i} \leq \sum_{\xi \in D \backslash D^{T-1}} v_{\xi}^{i}\left(\bar{Z}^{i}(\xi)\right.$ ) (this inequality follows the same arguments as Araujo, Páscoa and Torres-Martinez (2010), see Lemma B4 and Claim A2), so $\limsup _{T} \sum_{\xi: t(\xi)=T} \sum_{\eta \in \xi^{+}} L_{2 \eta}^{i}\left(\bar{Z}^{i}\right) \bar{Z}_{\xi}^{i} \leq$ 0 . Thus, by (18) and (19), $\limsup _{T}\left(\Pi^{i T}(Z)-\Pi^{i T}\left(\bar{Z}^{i}\right)\right) \leq \limsup _{T} \sum_{\xi: t(\xi)=T} L_{1 \xi}^{i}\left(\bar{Z}^{i}\right) Z_{\xi}$. Now,

$$
\begin{aligned}
L_{1 \xi}^{i}(\bar{Z}) Z_{\xi}= & \left(v_{\xi}^{i^{\prime}}(\bar{Z})-\mu^{i}(\xi) p(\xi)\right) x(\xi)-\mu^{i}(\xi) q(\xi) \theta(\xi) \\
& +\left(v_{\xi}^{i^{\prime}}(\bar{Z}) C(\xi)-\mu^{i}(\xi)(p(\xi) C(\xi)-q(\xi)) \varphi(\xi)\right. \\
& +\left(\tilde{\lambda}_{j}^{i}(\xi) d_{j}^{i}(\xi)+\rho_{j}(\xi)-\mu^{i}(\xi)\right) \Delta_{j}^{i}(\xi)
\end{aligned}
$$

where $v_{\xi}^{i^{\prime}}(\bar{Z})-\mu^{i}(\xi) p(\xi) \leq-\sum_{\eta \in \xi^{+}} \mu^{i}(\eta) p(\eta) Y(\eta) \leq 0$ and $\tilde{\lambda}_{j}^{i}(\xi) d_{j}^{i}(\xi)+\rho_{j}(\xi)-\mu^{i}(\xi)=0$

Proof of Theorem 1. Both along the sequence of finite economies equilibrium and at the limit point of the relevant subsequence, we have, by $(7)$, that $\lambda_{j}^{i}(\xi)<r_{\xi}^{i}(b(\xi))$ implies $\tilde{\lambda}_{j}^{i}(\xi) \leq \mu^{i}(\xi)$. It follows, by (4), that $\rho_{j}^{i}(\xi)>0$ and, therefore, $\Delta_{j}^{i}(\eta)=\operatorname{MIN}_{j}(\eta) \varphi_{j}^{i}(\xi)$. Suppose that for any agent (5) cannot hold with $d_{j}(\eta)=0, \forall \eta \in \xi^{+}$(otherwise we get immediately $p(\xi) C^{j}(\xi) \geq q^{j}(\xi)$, by (5)).

If promise $j$ is traded at $\xi$, we get $K^{j}(\eta)=\frac{\operatorname{MIN}_{j}(\eta)}{p(\eta) A^{j}(\eta)}$ for $\eta \in \xi^{+}$(along that subsequence and at its limit point) and (6) holds as equality for some agent. Combining with $(7)$, we get $p(\xi) C^{j}(\xi) \geq q^{j}(\xi)$, as for this agent we have:

$$
\mu^{i}(\xi)\left(p(\xi) C^{j}(\xi)-q^{j}(\xi)\right) \geq v_{\xi}^{\prime}\left(\bar{x}_{\xi}^{i}\right) C^{j}(\xi)+\sum_{\eta \in \xi^{+}} \mu^{i}(\eta)\left(p(\eta) Y(\eta) C^{j}(\xi)-M I N_{j}(\eta)\right) \geq 0
$$

If promise $j$ is not traded, but was traded along a subsequence (of the above subsequence), the above argument still applies. Otherwise, we can re-set $K^{j}(\eta)=\frac{\operatorname{MIN}_{j}(\eta)}{p(\xi) A^{j}(\xi)}$ (in fact, (6) remains true as we just lower the right hand side). Now, if (6) holds with strict inequality for every agent, with $K^{j}(\eta)=\frac{\operatorname{MIN}_{j}(\eta)}{p(\xi) A^{j}(\xi)}$, we lower $q^{j}(\xi)$, until $q^{j}(\xi)=\max _{i} \sum_{\eta \in \xi^{+}} \frac{\mu^{i}(\eta)}{\mu^{i}(\xi)} \operatorname{MIN}_{j}(\eta)$ (notice that (5) still holds, as we just raise the left-hand side). The agent(s) for whom this maximum occurs will have (20) satisfied and, therefore, $p(\xi) C^{j}(\xi) \geq q^{j}(\xi)$.

Actually the above resetting of $q^{j}(\xi), K^{j}(\eta)$ and $d_{j}(\eta)$ (for $\eta \in \xi^{+}$) when asset $j$ is not traded at node $\xi$, along any subsequence of truncated economies equilibria, could be done already along the relevant converging subsequence (rather than by modifying the limit point), so we are back in the exact setting addressed by the Proposition, knowing that $p(\xi) C^{j}(\xi) \geq q^{j}(\xi){ }^{8}$

Under (a), condition (8) in the Proposition holds. To see that it holds also under (b) or (c), notice that $v^{i^{\prime}}\left(\bar{Z}^{i}(\xi)\right) \leq \mu^{i}(\xi) p(\xi)$ and that $\sum_{\xi: t(\xi)=t} \mu^{i}(\xi) p(\xi) \omega^{i}(\xi) \longrightarrow 0$ as $t \longrightarrow \infty$. The latter follows as in Araujo, Páscoa and Torres-Martínez (2010). We have

$$
\begin{aligned}
-\sum_{\xi: t(\xi)=t} L_{1 \xi}^{i}\left(\bar{Z}^{i}(\xi), \bar{Z}^{i}\left(\xi^{-}\right)\right) Z^{i}(\xi) & \geq \sum_{\xi: t(\xi) \leq t}\left[L_{\xi}^{i}(0,0)-L_{\xi}^{i}\left(\bar{Z}^{i}(\xi), \bar{Z}^{i}\left(\xi^{-}\right)\right)\right] \\
& =\sum_{\xi: t(\xi) \leq t} \mu^{i}(\xi) p(\xi) \omega^{i}(\xi)-\Pi^{i t}\left(\bar{Z}^{i}\right),
\end{aligned}
$$

\footnotetext{
${ }^{8}$ In Section 5.2 we will address refinement concepts and see that this no-trade equilibrium, with expectations $K^{j}(\eta)=\frac{\operatorname{MIN}_{j}(\eta)}{p(\eta) A^{j}(\eta)}$ consistent with these moderate penalties, can be preserved under a refinement that removes both unduly pessimistic and unduly optimistic expectations.
} 
where $\limsup _{t}\left(-\sum_{\xi: t(\xi)=t} L_{1 \xi}^{i}\left(\bar{Z}^{i}(\xi), \bar{Z}^{i}\left(\xi^{-}\right)\right) Z^{i}(\xi)\right) \leq 0$ (as shown in the proof of the Proposition). So $\sum_{\xi} \mu^{i}(\xi) p(\xi) \omega^{i}(\xi) \leq \Pi^{i t}\left(\bar{Z}^{i}\right)<\infty$

Remark A.2: It can be seen from the proof of Theorem 1 that agents who have (6) holding with equality, for every promise, beyond some node $\xi$, will have (20) satisfied at these nodes for all promises and, therefore, have no opportunities for doing generalized Ponzi schemes.

\section{Proof of Theorem 2.}

(i)

For the finite horizon economy, we adapt the proof of Theorem 1 in Dubey, Geanakoplos and Shubik (2005). As in their proof the price set is $P_{\xi}=\left\{(p(\xi), q(\xi)): \sum_{g} p(\xi, g)=1, p(\xi, g) \geq s, q^{j}(\xi) \in\right.$ $[0,1 / s]\}$. Denote by $\psi_{s}^{0}$ the $\arg \max _{\xi \in D}\left\{\sum_{\xi \in D}\left(p(\xi) \cdot \sum_{i}\left(x^{i}(\xi)+\sum_{j \in J} C^{j}(\xi) \varphi_{j}^{i}(\xi)-W^{i}(\xi)\right)+q(\xi) \cdot \sum_{i}\left(\theta^{i}(\xi)-\right.\right.\right.$ $\left.\left.\left.\varphi^{i}(\xi)\right)\right)\right\}$ and by $K_{s}$ the $\operatorname{argmin}\left\{\sum_{\eta \in \xi^{+}}\left(\left(\sum_{i} \theta^{i}(\xi)\right) K_{s}^{j}(\eta) p(\eta) A^{j}(\eta)-\sum_{i} \Delta_{j}^{i}(\eta)\right)^{2}: K_{s}^{j}(\eta) \in[0,1], \forall \eta \in\right.$ $\left.\xi^{+}\right\}$.

Step 1. Now, we have to select the outcome that makes marginal penalty effects be dominated by marginal income effects. We do this by defining the correspondence $\psi_{s}^{\nu}(\xi)=\left\{\left(\nu_{\eta}\right)_{\eta \in \xi^{+}}=\nu \mathbb{l}\right.$ for some $\nu>$ $0: \sum_{\eta \in \xi^{+}} \nu_{\eta} \max _{i} \max \left\{\lambda_{j}^{i}(\eta), \mu^{i}(\eta)\right\} b_{\eta}^{j} \leq \sum_{\eta \in \xi^{+}} \min _{i} \min \left\{\lambda_{j}^{i}(\eta), \mu^{i}(\eta)\right\} p(\eta) Y(\eta) C^{j}(\eta), \forall j \in J^{*}$ and $\nu_{\eta} \in$ $\left.\left[0, \chi_{\xi}(s)\right]\right\}$, where $\chi_{\xi}(s)=\tilde{\chi}(s \mathbb{l}), \tilde{\chi}\left((p(\eta))_{\eta \in \xi^{+}}\right)=\frac{\max _{j} \sum_{\eta \in \xi^{+}} \min _{i} \min \left\{\lambda_{j}^{i}(\eta), \mu^{i}(\eta)\right\} p(\eta) Y(\eta) C^{j}(\xi)}{\min _{j} \sum_{\eta \in \xi^{+}} \max _{i} \max \left\{\lambda_{j}^{i}(\eta), \mu^{i}(\eta)\right\} b_{\eta}^{j}}$.

Step 2. We have to accommodate the nominal promises in the framework of the model with real promises. We do this by defining the mapping $\mathcal{A}_{\xi g}^{j}\left(j \in J^{*}\right)$ as the function $\left(\nu_{\xi}, b_{\xi}^{j}\right) \longmapsto \mathcal{A}_{\xi g}^{j}=\nu_{\xi} b_{\xi}^{j}$.

Step 3. Consumers have the standard constrained demand correspondence $\psi_{s}^{h}=\operatorname{argmax}_{Z_{\xi}}\left\{\Pi^{i}(Z):\right.$ budget and minimal repayment constraints hold at $(p, q, K)$, given $A$, for $Z=(x, \theta, \varphi, \Delta)$ such that $x(\xi) \leq \mathcal{W}_{\xi}(1+\delta), \varphi_{j}^{i}(\xi) \leq \frac{\mathcal{W}(1+\delta)}{\max _{g} C_{g}^{j}(\xi)} \equiv L_{\xi}^{j}, \theta_{j}^{i}(\xi) \leq(\# I) L_{\xi}^{j}, \Delta_{j}^{i}(\xi) \leq$ $\left.\left(\max _{g} A_{g}^{j}(\xi)\right) L_{\xi}^{j}\right)$, for some $\delta$ relatively small $\}$.

Step 4. Lagrange multipliers are obtained by introducing the correspondence $\boldsymbol{L}=\prod_{(i, \xi)} \boldsymbol{L}_{\xi}^{i}$ where $\boldsymbol{L}_{\xi}^{i}=\operatorname{argmin}_{\left(\mu^{i}(\xi), \rho_{j}(\xi)\right)}\left\{L_{\xi}^{i}\left(Z_{\xi}^{i}, Z_{\xi^{-}}^{i}, p(\xi), q(\xi), K(\xi), \mu^{i}(\xi), \rho(\xi)\right): \mu^{i}(\xi), \rho_{j}(\xi) \in\left[r_{\xi}^{i}, \bar{\mu}^{i}(\xi)\right]\right\}$.

Final step. For each $s>0$, a fixed point of $\psi_{s}^{0} \times K_{s} \times \psi_{s}^{\nu} \times A \times \boldsymbol{L} \times\left(\prod_{h} \psi^{h}\right)$ exists, as $\psi_{s}^{\nu}(\xi)$ is nonempty valued (take $\nu_{\eta}=\nu, \forall \eta \in \xi^{+}$, with $\left.\nu\left(\# \xi^{+}\right) \leq \frac{\min _{j \in J^{*}} \sum_{\eta \in \xi^{+}} \min _{i} \min \left\{\lambda_{j}^{i}(\eta), \mu^{i}(\eta)\right\} p(\eta) Y(\eta) C^{j}(\xi)}{\max _{j \in J^{*}} \sum_{\eta \in \xi^{+}} \max _{i} \max \left\{\lambda_{j}^{i}(\eta), \mu^{i}(\eta)\right\} b_{\eta}^{j}}\right)$ and upper hemicontinuous.

As in the proof of Theorem 1 in Dubey, Geanakoplos and Shubik (2005), when $s \longrightarrow 0$, aggregate excess demand goes to zero, $p(\eta, g)$ does not go to zero and $q(\xi)$ stays bounded. Moreover, $\nu_{\eta}$ stays both bounded from above and bounded away from zero. Passing to subsequences, we obtain a limit point which is an equilibrium for the finite horizon economy and satisfying condition (11), for any $(\eta, j) \in D \times J^{*}$, since at $\sigma_{\eta}=\lim \nu_{\eta}^{-1}$ we have (13) satisfied.

(ii) 
We redo the above argument, replacing $\psi_{s}^{\nu}(\xi)$ by $\tilde{\psi}_{s}^{\nu}(\xi)=\left\{\left(\nu_{\eta}\right)_{\eta \in \xi^{+}}=\nu \mathbb{I l}\right.$ for some $\nu>0$ :

$\sum_{\eta \in \xi^{+}} \max _{i} \max \left\{\lambda_{j}^{i}(\eta), \mu^{i}(\eta)\right\} p(\eta) A^{j}(\eta) \leq \sum_{\eta \in \xi^{+}} \nu_{\eta} \min _{i} \min \left\{\lambda_{j}^{i}(\eta), \mu^{i}(\eta)\right\} \tilde{y}_{\eta}^{j} C^{j}(\xi), \forall j \in J^{*}$ and $\nu_{\eta} \in$ $\left.\left[0, \hat{\chi}_{\xi}\right]\right\}$, where $\hat{\chi}_{\xi}=\frac{\left.\max _{j \in J^{*}} \sum_{\eta \in \xi^{+}} \max _{i} \max \left\{\lambda_{j}^{i}(\eta), \bar{\mu}^{i}(\eta)\right\} \bar{A}^{j}(\eta)\right)}{\min _{j \in J^{*}} \sum_{\eta \in \xi^{+}} \min _{i} \min \left\{\lambda_{j}^{i}(\eta), r^{i}(\eta)\right\} \tilde{y}_{\eta}^{j} C^{j}(\xi)}$.

We also replace the construction of real returns matrices for the promises by those for the collateral: let $Y_{\xi g}^{j}\left(j \in J^{*}\right)$ be the function $\left(\nu_{\xi}, \tilde{y}_{\xi}^{j}\right) \longmapsto Y_{\xi g}^{j}=\nu_{\xi} \tilde{y}_{\xi}^{j}$. Notice that $\tilde{\psi}_{s}^{\nu}(\eta)$ is nonempty valued (take $\left.\sigma \geq \frac{\left.\max _{j \in J^{*}} \sum_{\eta \in \xi^{+}} \max _{i} \max \left\{\lambda_{j}^{i}(\eta), \mu^{i}(\eta)\right\} p(\eta) A^{j}(\eta)\right)}{\min _{j \in J^{*}} \sum_{\eta \in \xi^{+}} \min _{i} \min \left\{\lambda_{j}^{i}(\eta), \mu^{i}(\eta)\right\} \tilde{y}_{\eta}^{j} C^{j}(\xi)}\right)$ and upper hemicontinuous. The correspondence $\psi_{s}^{0} \times K_{s} \times$ $\tilde{\psi}_{s}^{\nu} \times Y \times \psi^{\prime} \times\left(\prod_{l} \psi^{l}\right)$ has a fixed point.

A cluster point, as $s \longrightarrow 0$, of the sequence of fixed points is an equilibrium for the finite horizon economy. Notice that, along the converging subsequence, $\nu_{\eta}$ is bounded away from zero and take $\sigma_{\eta}=\lim \nu_{\eta}$. At the limit, (14) holds.

(iii)

Step $A$. Let $\hat{J}$ be the subset of $J^{*}$ consisting of nominal promises backed by nominal collateral with endogenous coefficients $\vartheta_{g(j)}^{j}(\xi)$. Denote by $\hat{\psi}^{\nu}=\left\{\left(\nu_{\eta}\right)_{\eta \in \xi^{+}} \in \Delta^{\# \xi^{+-1}}\right.$ and $\vartheta_{g(j)}^{j}(\xi) \geq 0, j \in$ $\hat{J}: \sum_{\eta \in \xi^{+}: \tilde{y}^{j}(\eta)>0} \nu_{\eta(j)} \min _{i} \min \left\{\lambda_{j}^{i}(\eta(j)), \mu^{i}(\eta(j))\right\} \tilde{y}_{\eta(j)}^{j} \geq \sum_{\eta \in \xi^{+}} \max _{i} \max \left\{\lambda_{j}^{i}(\eta), \mu^{i}(\eta)\right\} b_{\eta}^{j} \vartheta_{g(j)}^{j}(\xi)$, for $j \in$ $\hat{J}\}$. This correspondence is nonempty valued: we can always take $\eta(j): \tilde{y}^{j}(\eta(j))>0$, make $\nu_{\eta(j)}=$ $\vartheta_{g(j)}^{j}(\xi) \frac{\sum_{\eta(j)} \max _{i} \max \left\{\lambda_{j}^{i}(\eta), \mu^{i}(\eta)\right\} b_{\eta}^{j}}{\min \min \left\{\lambda_{j}^{i}(\eta(j)), \mu^{i}(\eta(j))\right\} \tilde{y}_{\eta}^{j}(j)} \equiv R_{\eta(j)}^{j}\left(\vartheta_{g(j)}^{j}(\xi)\right)$ and choose $\left(\vartheta_{g(j)}^{j}(\xi)\right)$ high enough so that $\sum_{j \in \hat{J}} R_{\eta(j)}^{j}\left(\vartheta_{g(j)}^{j}(\xi)\right) \leq 1$

Step B. Now, let $J_{1}$ be the subset of $J^{*}$ consisting of nominal promises backed by real collateral. Let $\psi_{1 s}^{\nu}=\left\{\left(\nu_{\eta}\right)_{\eta \in \xi^{+}} \in \Delta^{\# \xi^{+}-1}\right.$ and $\alpha_{\xi}^{j} \geq 0, j \in J_{1}: \sum_{\eta(j): b_{\eta}^{j}>0} \nu_{\eta(j)} \max _{i} \max \left\{\lambda_{j}^{i}(\eta(j)), \mu^{i}(\eta(j))\right\} b_{\eta}^{j} \leq$ $\sum_{\eta \in \xi^{+}} \min _{i} \min \left\{\lambda^{i}(\eta), \mu^{i}(\eta)\right\} p(\eta) Y(\eta) \alpha_{\xi}^{j} C^{j}(\xi)-\sum_{\eta: b_{\eta}^{j}=0} \max _{i} \max \left\{\lambda_{j}^{i}(\eta(j)), \mu^{i}(\eta(j))\right\} b_{\eta}^{j}$, for $\left.j \in J_{1},\right\}$. This correspondence is nonempty valued: by scaling up $C^{j}(\xi)$ by $\alpha_{\xi}^{j}$, high enough, we can make the right hand side in these inequalities positive (as $p(\eta, g) \geq s, \forall \eta, \forall g$ ).

Step C. Actually, $\hat{\psi}^{\nu} \cap \psi_{1 s}^{\nu}$ is nonempty valued. Moreover, $\hat{\psi}^{\nu}$ takes uniformly bounded values in $\gamma_{g(j)}^{j}(\xi)$ and $\psi_{1 s}^{\nu}$ takes uniformly bounded values in $\alpha_{\xi}^{j} \cdot{ }^{9}$ We can even see that $\hat{\psi} \equiv \hat{\psi}^{\nu} \cap\left\{\left(\nu_{\eta}\right)_{\eta \in \xi^{+}}\right.$: $\left.\sum_{\eta(j), j \in \hat{J}} \nu_{\eta(j)} \leq 1-\epsilon\right\}$ is nonempty, for $\epsilon$ small enough (as $\gamma_{g(j)}^{j}(\xi)$ can accommodate this requirement) and we let $\tilde{\nu}_{\xi}$ be the function $\left(\nu_{\eta(j)}\right)_{j \in \hat{J}} \longmapsto\left(\nu_{\eta}\right)_{\eta \in \xi^{+}}$, where $\nu_{\eta}=\frac{1-\sum_{j \in \hat{J}} \nu_{\eta(j)}}{\# \xi^{+}-\# \hat{J}}$ for $\eta \neq \eta(j), j \in \hat{J}$. Denote by $\psi_{s}^{N}$ the nonempty intersection $\hat{\psi} \cap \psi_{1 s}^{\nu} \cap\left\{\tilde{\nu}_{\xi}\right\}$.

The correspondence $\psi_{s}^{0} \times K_{s} \times \psi_{s}^{N} \times Y \times \mathcal{A} \times \psi^{1} \times\left(\prod_{h} \psi^{h}\right)$ has a fixed point. As $s \longrightarrow 0, \nu_{\eta}$ stays bounded and bounded away from zero. Taking a convergent subsequent of fixed points, we let $\sigma_{n}=\lim \nu_{n}^{-1}$ and we see that the limit of the fixed points is an equilibrium for the finite horizon economy where (13) and (15) hold (respectively, for a nominal-real contract and for a nominal-nominal

\footnotetext{
${ }^{9}$ The choice set for $\alpha_{\xi}^{j}, j \in J_{1}$, is bounded by $\frac{\sum_{\eta: b_{\eta}^{j}=0} \max \max \left\{\lambda_{j}^{i}(\eta), \bar{\mu}^{i}(\eta)\right\} b_{\eta}^{j}}{\sum_{\eta \in \xi} \min _{i} \min \left\{\lambda_{j}^{i}(\eta), r^{i}(\eta)\right\} s \sum_{l, g} Y_{\eta g}^{l} \tilde{c}_{g}^{j}(\xi)}$. The choice set for $\vartheta_{g(j)}^{j}(\xi), j \in \hat{J}$, is bounded by $\frac{\min _{i} \min \left\{\lambda_{j}^{i}(\eta(j)), r^{i}(\eta)\right\} \tilde{y}_{\eta(j)}}{\sum_{\eta \in \xi^{+}} \max _{i} \max \left\{\lambda_{j}^{i}(\eta), \bar{\mu}^{i}(\eta)\right\} b_{\eta}^{j}}$, for some $\eta(j): \tilde{y}^{j}(\eta(j))>0$.
} 
contract).

We omit the less interesting case of real promises backed by nominal collateral (but the argument for the set $J_{2}$ of such contracts follows closely the one made for $\hat{J}$.

Proof of Claim 5.4. We adapt the proof of Theorem 1 in Dubey, Geanakoplos and Shubik (2005). As in that proof, for each $s$, the price set is such that $p(\xi, g) \geq s, \sum_{g} p(\xi, g)=1$ and $q^{j}(\xi) \leq \frac{1}{s}$. We define the function $F$ mapping each vector $\left(\tilde{x}^{i}(\xi, g(j))\right)_{i}$ into the value $F(\xi, g(j))$ equal to $\min _{i}\left\{\tilde{x}^{i}(\xi, g(j))\right)$ : $\left.\tilde{x}^{i}(\xi, g(j))>0\right\}$ if $\left(\tilde{x}^{i}(\xi, g(j))\right)_{i} \neq 0$ and equal to zero otherwise. It is a continuous function. Now, for each $i$, let $\bar{\beta}_{j}^{i}(\xi)=\frac{\left.\tilde{x}^{i}(\xi, g(j))\right)}{s+F(\xi, g(j))}$.

Define also the following correspondences: $G_{j}^{i}(\xi)=\operatorname{argmax}\left\{\gamma_{j}^{i}(\xi)\left(\tilde{\lambda}_{j}^{i}(\xi)-\mu^{i}(\xi)\right): \gamma_{j}^{i}(\xi) \in[0,1]\right\}$ and let $\tilde{G}_{j}^{i}(\xi)=\operatorname{argmin}\left\{\left[\zeta_{j}^{i}(\xi) p(\xi) A^{j}(\xi)-\gamma_{j}^{i}(\eta) p(\eta) A^{j}(\eta)-\left(1-\gamma_{j}^{i}(\eta)\right) \operatorname{MIN}_{j}(\eta)\right]^{2}: \zeta_{j}^{i}(\xi) \in[0,1]\right\}$.

Delivery rates $K^{j}(\eta)$ are defined jointly with weights $\beta_{j}^{i}(\xi)$ by the next correspondence. The conditions this correspondence imposes on the weights $\beta_{j}^{i}(\xi)$ become redundant when the promise is traded. For each $(\xi, j):\left(\left(K^{j}(\eta)_{\eta \in \xi^{+}},\left(\beta_{j}^{i}\right)_{i}\right) \in \boldsymbol{H}_{\xi}^{j}\right.$, where

$$
\begin{aligned}
\boldsymbol{H}_{\xi}^{j} \equiv & \operatorname{argmin}\left\{\sum _ { \eta \in \xi ^ { + } } \left[\left(\left(\sum_{i} \theta_{j}^{i}(\xi)\right) K^{j}(\eta) p(\eta) A^{j}(\eta)-\sum_{i} \Delta_{j}^{i}(\eta)\right)^{2}+\left(K^{j}(\eta)-\sum_{i} \beta_{j}^{i}(\xi) \zeta_{j}^{i}(\eta)\right)^{2}\right.\right. \\
+ & \sum_{i}\left(\alpha_{j}^{i}(\xi) \beta_{j}^{i}(\xi)+\beta_{j}^{i}(\xi)\left[\mu^{i}(\eta)-\tilde{\lambda}_{j}^{i}(\eta)\right]^{+}\left(1-d_{j}^{i}(\eta)\right)+\left(\beta_{j}^{i}(\xi) \sum_{k} \varphi_{j}^{k}(\xi)-\varphi_{j}^{i}(\xi)\right)^{2}\right): K^{j}(\eta) \in[0,1] \\
& \left.\sum_{i} \beta_{j}^{i}(\xi) \leq \frac{1}{1+s}, 0 \leq \beta_{j}^{i}(\xi) \leq \bar{\beta}_{j}^{i}(\xi)\right\}
\end{aligned}
$$

where $\alpha_{j}^{i}(\xi)$ is the slack in $(5)$.

Lagrange multipliers $\left(\mu^{i}(\xi), \rho^{i}(\xi)\right)$ are given by $\boldsymbol{L}_{\xi}^{i} \equiv \operatorname{argmin}\left\{L_{\xi}^{i}\left(x^{i}, \varphi^{i}, \theta^{i}, \Delta^{i}, p, q, K\right): \mu^{i}(\xi) \in\right.$ $\left.\left[0, \bar{\mu}^{i}(\xi)\right], \rho^{i}(\xi) \in\left[0, \bar{\mu}^{i}(\xi)\right]\right\}$ and the supergradients $d_{j}^{i}(\eta)$ are given by $D_{j}^{i}(\eta) \equiv \operatorname{argmin}\left\{\left(\tilde{\lambda}_{j}^{i}(\eta) d_{j}^{i}(\eta)-\mu^{i}(\eta)\right)^{2}\right.$ : $\left.d_{j}^{i} \in \partial(+)\left(p(\eta) A^{j}(\eta) \varphi_{j}^{i}(\eta)-\Delta_{j}^{i}(\eta)\right)\right\}$, where + stands for the function $z \longmapsto z^{+}$.

For each $s$, a fixed point of $\psi_{s}^{0} \times F \times \bar{\beta} \times \prod_{(j, \xi)} \boldsymbol{H}_{\xi}^{j} \times \prod_{i} \psi_{s}^{i} \times \prod_{i, \xi} \boldsymbol{L}_{\xi}^{i} \times \prod_{(i, \xi, j)} \boldsymbol{D}_{j}^{i}(\xi) \times \prod_{(i, \xi, j)} G_{j}^{i}(\xi) \times \prod_{(i, \xi, j)} \tilde{G}_{j}^{i}(\xi)$ exists.

As $s \longrightarrow 0$, the sequence of fixed points has a cluster point, at which market clearing holds, $p(\xi, g)$ does not converge to zero and $q^{j}(\xi)$ stays bounded. This cluster point is an equilibrium $E$ for the finite horizon economy such that if $j \in \tilde{J}_{\xi}(E), K^{j}(\eta)=\sum_{i} \beta_{j}^{i}(\xi) \zeta_{j}^{i}(\eta)$ where $\beta_{j}^{i}(\xi)=0$ for $i$ with $x^{i}(\xi, g(j))=0$ or $\alpha_{j}^{i}(\xi)=0$. Moreover, if $V_{\xi}^{j}(E) \neq \emptyset$, then $\beta_{j}^{i}(\xi)>0$ only for $i \in V_{\xi}^{j}(E)$.

\section{REFERENCES.}

Araujo, A.P., Páscoa, M.R., Torres-Martínez, J.P., 2002. Collateral avoids Ponzi schemes in incomplete markets. Econometrica, 70, 1613-1638.

Araujo, A.P., Páscoa, M.R., Torres-Martínez, J.P., 2010. Long Lived Collateralized Assets and Bubbles, Journal of Mathematical Economics, forthcoming.

Dubey, Pr., Geanakoplos, J., Shubik, M., 2005. Default and Punishment in General Equilibrium. Econometrica, 73, 1-37.

Fostel, A., Geanakoplos, J., 2008. Leverage Cycles and the Anxious Economy. American Economic Review, 98(4): 1211-44. 
Kubler, F., Schmedders, K., 2003. Stationary equilibria in asset-pricing models with incomplete markets and collateral. Economterica 71, 1767-1793.

Magill, M., Quinzii, M., 1996. Incomplete Markets over an Infinite Horizon: Long-lived Securities and Speculative Bubbles. Journal of Mathematical Economics, 26, 133-170.

Martins-da-Rocha, V.F., Vailakis, Y., 2010. On Ponzi schemes in infinite horizon collateralized economies with default penalties, presented at the IV Vigo Workshop on Frontiers in Economic Theory and Applications, Baiona, September 13-14, 2010.

Páscoa, M.R., Seghir, A., 2009. Harsh default penalties lead to Ponzi schemes. Games and Economic Behavior, 65, 270-286. 